\title{
THE COHOMOLOGY OF REAL DE CONCINI-PROCESI MODELS OF COXETER TYPE
}

\author{
ANTHONY HENDERSON AND ERIC RAINS
}

\begin{abstract}
We study the rational cohomology groups of the real De Concini-Procesi model corresponding to a finite Coxeter group, generalizing the type- $A$ case of the moduli space of stable genus 0 curves with marked points. We compute the Betti numbers in the exceptional types, and give formulae for them in types $B$ and $D$. We give a generating-function formula for the characters of the representations of a Coxeter group of type $B$ on the rational cohomology groups of the corresponding real De Concini-Procesi model, and deduce the multiplicities of one-dimensional characters in the representations, and a formula for the Euler character. We also give a moduli space interpretation of this type- $B$ variety, and hence show that the action of the Coxeter group extends to a slightly larger group.
\end{abstract}

\section{INTRODUCTION}

In [6, the second author and his collaborators studied the rational cohomology ring of $\overline{\mathcal{M}_{0, n}}(\mathbb{R})$, the manifold of real points of the moduli space of stable genus 0 curves with $n$ marked points. In [14], the second author described this ring as a representation of $S_{n}$, giving a formula for the graded character. The crux of both papers was a close connection between the cohomology of $\overline{\mathcal{M}_{0, n}}(\mathbb{R})$ and the homology of the poset of partitions of $\{1,2, \cdots, n-1\}$ into parts of odd size. One explanation for this connection is that $\overline{\mathcal{M}_{0, n}}(\mathbb{R})$ can be regarded as the real De Concini-Procesi model of the hyperplane arrangement of type $A_{n-2}$; in this context the relation with poset homology was generalized to an arbitrary subspace arrangement in [15]. It was then natural to try to extend the results of [6] and [14 to the other finite Coxeter types, and that is the goal of the present work.

For any irreducible finite Coxeter group $W$ with reflection representation $V$ over $\mathbb{R}$, the real De Concini-Procesi model $\bar{Y}_{W}(\mathbb{R})$ is defined

This work was supported by Australian Research Council grant DP0344185. 
(see Section 1) to be the closure of the image of the map

$$
M_{W} \rightarrow \prod_{W^{\prime} \in \Pi_{W}^{\mathrm{irr}}} \mathbb{P}\left(V / V^{W^{\prime}}\right)
$$

where $M_{W}$ is the complement of the reflecting hyperplanes and $\Pi_{W}^{\mathrm{irr}}$ denotes the set of irreducible parabolic subgroups of $W$. This is a nonsingular projective real variety. If $W=S_{n-1}, \bar{Y}_{W}(\mathbb{R})$ is isomorphic to $\overline{\mathcal{M}_{0, n}}(\mathbb{R})$, which has been studied in [5] and [3] as well as the papers mentioned above. The general Coxeter case has been considered in [3], [7], and [1], from varying points of view and with different emphases from the present paper, which concentrates on the rational cohomology groups $H^{i}\left(\bar{Y}_{W}(\mathbb{R}) ; \mathbb{Q}\right)$. The cohomology groups of the corresponding complex varieties have also been studied - see, for instance, [8] for $\overline{\mathcal{M}_{0, n}}(\mathbb{C}),[18$ for the classical types, and [9] for type $B-$ but these behave very differently from the real case.

In Section 1 of this paper we recall the main result of [15], expressing the cohomology of a real De Concini-Procesi model in terms of the homology of a corresponding poset: in the case of $\bar{Y}_{W}(\mathbb{R})$, the poset $\Pi_{W}^{(2)}$ consists of those parabolic subgroups of $W$ whose irreducible components all have even rank. We prove in Theorem 1.5 that this poset is Cohen-Macaulay, and deduce in Theorem 1.6 that $H^{i}\left(\bar{Y}_{W}(\mathbb{R}) ; \mathbb{Q}\right)$ coincides with the $i$ th Whitney homology $W H_{i}\left(\Pi_{W}^{(2)}\right)$, up to some twisting by sign representations. We also prove a result (Corollary 1.8) reducing most questions in type $D$ to type $B$.

In Section 2 we compute the Poincaré polynomial and Euler characteristic of $\bar{Y}_{W}(\mathbb{R})$ (these equal the characteristic polynomial and Euler characteristic of the poset $\left.\Pi_{W}^{(2)}\right)$. This section is brief, since type $A$ was done in [6], type $B$ was effectively done in [10] (though the Poincaré polynomial formula is not as explicit as in type $A$ ), type $D$ reduces to type $B$, and the other types can be calculated directly. (The Euler characteristics in the classical types were calculated in [5], [7], and [1]).

In Theorem 3.2 we prove a plethystic generating-function formula for the characters of the representations of $W\left(B_{n}\right)$ on $H^{i}\left(\bar{Y}_{B_{n}}(\mathbb{R}) ; \mathbb{Q}\right)$. This is analogous to the type- $A$ result in [14], but does not give as explicit a formula for the graded character of a particular group element. However, we are able to deduce the multiplicities of the one-dimensional characters (Proposition 3.5), as well as a formula for the Euler character (Proposition 3.7).

In Theorem 4.1 we give a moduli space intepretation of the variety $\bar{Y}_{B_{n}}$ : it can be embedded as a closed subvariety in $\overline{\mathcal{M}_{0,2 n+2}}$, namely the one parametrizing stable curves possessing an involution which fixes 
the last two marked points, interchanges the $j$ th and $(n+j)$ th for $1 \leq$ $j \leq n$, and does not fix any component pointwise. Swapping the labels of the last two marked points induces an involution $\sigma$ of $\bar{Y}_{B_{n}}$ which commutes with the $W\left(B_{n}\right)$-action, and we give some partial results about the action of this involution on the cohomology of $\bar{Y}_{B_{n}}(\mathbb{R})$.

\section{Real De Concini-Procesi models of Coxeter type}

Let $V$ be a finite-dimensional real vector space, and $\mathcal{G}$ a building set in the dual space $V^{*}$. (See [4] for the definition of building set, and of the building set(s) associated to a subspace arrangement in $V$.) For any $G \in \mathcal{G}$, let $G^{\perp}$ denote the orthogonal subspace of $V$, and let $M_{\mathcal{G}}$ be the complement $V \backslash \bigcup_{G \in \mathcal{G}} G^{\perp}$. The associated (compact) real De Concini-Procesi model $\bar{Y}_{\mathcal{G}}(\mathbb{R})$ is the closure of the image of the map

$$
M_{\mathcal{G}} \rightarrow \prod_{G \in \mathcal{G}} \mathbb{P}\left(V / G^{\perp}\right)
$$

induced by the natural maps $M_{\mathcal{G}} \rightarrow\left(V / G^{\perp}\right) \backslash\{0\} \rightarrow \mathbb{P}\left(V / G^{\perp}\right)$. Here it makes no difference whether the closure is taken in the Zariski or in the usual topology, because Gaiffi's result [7, Theorem 4.1] shows that the topological closure coincides with the set of real points of the variety $\bar{Y}_{\mathcal{G}}$ defined using Zariski closure. It was shown in 4 that $\bar{Y}_{\mathcal{G}}$ is a nonsingular projective variety, and hence $\bar{Y}_{\mathcal{G}}(\mathbb{R})$ is a compact smooth manifold, which is easily seen to be connected.

Let $\Pi_{\mathcal{G}}$ denote the lattice of all subspaces of $V^{*}$ which can be written as direct sums of elements of $\mathcal{G}$, and write $\Pi_{\mathcal{G}}^{(2)}$ for the sub-poset consisting of direct sums of even-dimensional elements of $\mathcal{G}$. In [15, Theorem 3.7] the second author expressed the homology groups of $\bar{Y}_{\mathcal{G}}(\mathbb{R})$ (modulo 2-torsion) in terms of the poset cohomology groups of $\Pi_{\mathcal{G}}^{(2)}$. The rational cohomology groups of $\bar{Y}_{\mathcal{G}}$ can be similarly expressed in terms of the rational homology groups of $\Pi_{\mathcal{G}}^{(2)}$. For any $A \in \Pi_{\mathcal{G}}^{(2)} \backslash\{0\}$, let $H_{i}(0, A)$ denote the reduced poset homology $\widetilde{H}_{i-2}((0, A) ; \mathbb{Q})$ of the open interval $(0, A)$, and define $H_{i}(0,0)$ to be $\mathbb{Q}$ if $i=0$ and 0 otherwise. Also let $\operatorname{or}(A)$ denote $\widetilde{H}_{\operatorname{dim} A-1}(\mathbb{P} A ; \mathbb{Q})$; since $\operatorname{dim} A$ is even, this is a one-dimensional vector space on which $G L(A)$ acts via the sign of the determinant.

Theorem 1.1. For all $i$, there is an isomorphism of $\mathbb{Q}$-vector spaces

$$
H^{i}\left(\bar{Y}_{\mathcal{G}}(\mathbb{R}) ; \mathbb{Q}\right) \cong \bigoplus_{A \in \Pi_{\mathcal{G}}^{(2)}} H_{\operatorname{dim} A-i}(0, A) \otimes \operatorname{or}(A)
$$

which is equivariant for the subgroup of $G L(V)$ preserving $\mathcal{G}$. 
Proof. Apply $\operatorname{Hom}_{\mathbb{Z}}(-, \mathbb{Q})$ to the isomorphism in [15, Theorem 3.7]. Equivariance follows from the naturality shown there.

Observe the similarity between this result and that of Goresky and MacPherson (equivariantly, Sundaram and Welker - see [16, Section 5.4]) relating the cohomology of $M_{\mathcal{G}}$ to the homology of the lattice $\Pi_{\mathcal{G}}$. In this paper we consider building sets associated to hyperplane arrangements of finite Coxeter type. Let $\mathcal{A}$ be such a hyperplane arrangement in $V$, which we may as well assume to be essential (i.e. the intersection of all the hyperplanes is 0 ), and let $W$ be the corresponding finite Coxeter group. We write $n$ for $\operatorname{rk}(W)=\operatorname{dim} V$. For any hyperplane $H \in \mathcal{A}$, let $\alpha_{H} \in V^{*}$ be a linear form such that $H=\operatorname{ker} \alpha_{H}$. Let $\mathcal{G}$ be the minimal building set associated to $\mathcal{A}$, namely the set of all subspaces $G \subseteq V^{*}$ which are spanned by some subset of the $\alpha_{H}$ 's, and cannot be written as a nontrivial direct sum $G=G_{1} \oplus G_{2}$ in such a way that every $\alpha_{H} \in G$ is contained in either $G_{1}$ or $G_{2}$. Then $\Pi_{\mathcal{G}}$ is the lattice of all subspaces spanned by a subset of the $\alpha_{H}$ 's.

It is well known that $\Pi_{\mathcal{G}}$ is isomorphic to the lattice $\Pi_{W}$ of parabolic subgroups of $W$, via the bijection sending a parabolic subgroup $W^{\prime}$ to the subspace $\left(V^{W^{\prime}}\right)^{\perp} \subseteq V^{*}$, whose dimension is $\operatorname{rk}\left(W^{\prime}\right)$. (Note that $\Pi_{W}$ contains all parabolic subgroups, not just the standard parabolic subgroups relative to some chosen Coxeter system.) Under this bijection $\mathcal{G}$ corresponds to the set $\Pi_{W}^{\mathrm{irr}}$ of irreducible parabolic subgroups, and the sub-poset $\Pi_{\mathcal{G}}^{(2)}$ corresponds to the poset $\Pi_{W}^{(2)}$ of parabolic subgroups of $W$ all of whose irreducible components have even rank. The minimum element of $\Pi_{W}^{(2)}$ will be written $\hat{0}$, although it is just the trivial subgroup; if all irreducible components of $W$ have even rank, there is also a maximum element in $\Pi_{W}^{(2)}$, namely $W$.

Thus we can rephrase the definition of $\bar{Y}_{W}=\bar{Y}_{\mathcal{G}}$ as follows: it is the closure of the image of the map

$$
M_{W} \rightarrow \prod_{W^{\prime} \in \Pi_{W}^{\mathrm{irr}}} \mathbb{P}\left(V / V^{W^{\prime}}\right)
$$

where $M_{W}=V \backslash \bigcup_{H \in \mathcal{A}} H$ is the hyperplane complement. The normalizer $N_{G L(V)}(W)$ preserves $\mathcal{G}$ and acts on $\bar{Y}_{W}$ by variety automorphisms. The manifold $\bar{Y}_{W}(\mathbb{R})$ is closely related to the minimal blow-up of the Coxeter complex, studied in [3] and [1] (among other papers); the latter could be obtained by including in the codomain of (1.1) the factor $S(V)$, the unit sphere in $V$. (If $W$ is irreducible, the minimal blow-up of the Coxeter complex is a double cover of $\bar{Y}_{W}(\mathbb{R})$.) Our aim here is to describe the rational cohomology of $\bar{Y}_{W}(\mathbb{R})$, using Theorem 1.1 . 
It is clear that if $W \cong W_{1} \times W_{2}$ is reducible, then $\Pi_{W} \cong \Pi_{W_{1}} \times \Pi_{W_{2}}$ and $\bar{Y}_{W} \cong \bar{Y}_{W_{1}} \times \bar{Y}_{W_{2}}$, so we can restrict attention to the case that $W$ is irreducible. In this case the image of $M_{W} \rightarrow \bar{Y}_{W}$ is isomorphic to the image $\mathbb{P} M_{W}$ of $M_{W} \rightarrow \mathbb{P}(V)$, which implies that $\operatorname{dim} \bar{Y}_{W}=n-1$. Note that if $n=1$, then $\bar{Y}_{W}$ is a point, and if $n=2$, then $\bar{Y}_{W}$ is a projective line, so the interest lies in rank $\geq 3$. When speaking of a particular type $X_{n}$, we will write $W$ as $W\left(X_{n}\right), \Pi_{W}$ as $\Pi_{X_{n}}, \bar{Y}_{W}$ as $\bar{Y}_{X_{n}}$, and so forth.

The crucial question is what special properties the poset $\Pi_{W}^{(2)}$ has that a general $\Pi_{\mathcal{G}}^{(2)}$ does not. We first consider the classical types.

Example 1.2. Suppose $W=W\left(A_{n}\right) \cong S_{n+1}$. Then $\Pi_{A_{n}}$ is isomorphic to the partition lattice $\Pi_{n+1}$, and $\Pi_{A_{n}}^{(2)}$ corresponds to the poset $\Pi_{n+1}^{1 \bmod 2}$ of partitions of $\{1, \cdots, n+1\}$ into parts of odd size. This poset was shown to be Cohen-Macaulay by Björner, and its Möbius function was studied in [2].

Example 1.3. Suppose $W=W\left(B_{n}\right) \cong\{ \pm 1\}<S_{n}$. Then $\Pi_{B_{n}}$ is isomorphic to the Dowling lattice $Q_{n}(\{ \pm 1\}$ ) (also known as the signed partition lattice). Following [10], this consists of pairs $(J, \pi)$ where $J=\{ \pm 1\} \times I$ for some subset $I \subseteq\{1, \cdots, n\}$ and $\pi$ is a partition of $(\{ \pm 1\} \times\{1, \cdots, n\}) \backslash J$ whose parts are interchanged in pairs by the action of $\{ \pm 1\}$. The sub-poset $\Pi_{B_{n}}^{(2)}$ corresponds to the sub-poset of $Q_{n}(\{ \pm 1\})$ defined by the conditions $\frac{|J|}{2} \equiv 0 \bmod 2$ and $|K| \equiv 1$ $\bmod 2$ for all $K \in \pi$; this is the sub-poset denoted $Q_{n}^{1} \bmod 2(\{ \pm 1\})$ in [10, Definition 1.3], except that when $n$ is odd we must remove the maximum element artifically added to make the latter poset bounded. So [10, Proposition 1.4] shows that $\Pi_{B_{n}}^{(2)}$ is Cohen-Macaulay.

Example 1.4. Suppose $W=W\left(D_{n}\right)$ for $n \geq 4$. There is an obvious poset embedding $\Pi_{D_{n}}^{(2)} \hookrightarrow \Pi_{B_{n}}^{(2)}$ which replaces any $D_{\ell}$ factor of a parabolic subgroup by a $B_{\ell}$ factor. The image of this embedding is the sub-poset defined by the condition $\frac{|J|}{2} \neq 2$ (because $D_{2}=A_{1} \times A_{1}$ is reducible). One could prove Cohen-Macaulayness of this sub-poset by an argument similar to [10, Proposition 1.4], although there is a slight complication: the principal upper order ideals corresponding to pairs $(\emptyset, \pi)$ are not in general isomorphic to a smaller $\Pi_{D_{\ell}}^{(2)}$, but rather to posets associated to the hyperplane arrangements interpolating between types $B$ and $D$ (i.e. those denoted $\mathcal{A}_{\ell}^{k}$ in [13, Proposition 6.82]).

We have the following type-independent result (see [16] for the definitions involved). Note that finiteness of $W$ is necessary only for (3). 
Theorem 1.5. Let $W$ be a finite Coxeter group.

(1) $\Pi_{W}^{(2)}$ is pure with rank function $\mathrm{rk}_{\Pi_{W}^{(2)}}\left(W^{\prime}\right)=\frac{\mathrm{rk}\left(W^{\prime}\right)}{2}$.

(2) If $W$ is irreducible of even rank, then $\Pi_{W}^{(2)}$ is semimodular; if $W$ is irreducible of odd rank, then $\Pi_{W}^{(2)} \cup\{\hat{1}\}$ is semimodular.

(3) $\Pi_{W}^{(2)}$ is Cohen-Macaulay.

Proof. Let $I$ be the set of vertices of the Coxeter graph of $W$. For any $J \subseteq I$, let $W_{J}$ denote the corresponding standard parabolic subgroup. It is well known that every chain of parabolic subgroups of $W$ is $W$ conjugate to a chain of standard parabolic subgroups.

To prove (1), it suffices to show that if $W^{\prime} \subset W^{\prime \prime}$ is a cover relation in $\Pi_{W}^{(2)}$, then $\operatorname{rk}\left(W^{\prime \prime}\right)=\operatorname{rk}\left(W^{\prime}\right)+2$. Conjugating by a suitable element of $W$ if necessary, we can assume that $W^{\prime}=W_{J}$ and $W^{\prime \prime}=W_{K}$ are standard parabolic subgroups, where $J \subset K$. Regarding $J$ and $K$ as subgraphs of the Coxeter graph of $W$, we know by definition of $\Pi_{W}^{(2)}$ that every connected component of $K$ is even (i.e. has an even number of vertices), and similarly for $J$. Moreover, this evenness property does not hold for any subgraph $J^{\prime}$ such that $J \subset J^{\prime} \subset K$. It is easy to see that this forces $|K \backslash J|=2$ as required.

In part (2), we assume that $W$ is irreducible, so the Coxeter graph is connected. We must show that if $W^{\prime}, W^{\prime \prime} \in \Pi_{W}^{(2)}$ are different covers of a single element $W^{0}$, then either there is another element of $\Pi_{W}^{(2)}$ which covers $W^{\prime}$ and $W^{\prime \prime}$, or both $W^{\prime}$ and $W^{\prime \prime}$ are maximal in $\Pi_{W}^{(2)}$ (the latter case being possible only if $\operatorname{rk}(W)$ is odd). By part (1), the assumption implies that $\operatorname{rk}\left(W^{\prime}\right)=\operatorname{rk}\left(W^{\prime \prime}\right)=\operatorname{rk}\left(W^{0}\right)+2$. Hence the join $\left\langle W^{\prime}, W^{\prime \prime}\right\rangle$ in the geometric lattice $\Pi_{W}$ has rank either $\operatorname{rk}\left(W^{0}\right)+3$ or $\operatorname{rk}\left(W^{0}\right)+4$. Conjugating by a suitable element of $W$, we can assume that $W^{0}=W_{J}$, $W^{\prime}=W_{K}$, and $\left\langle W^{\prime}, W^{\prime \prime}\right\rangle=W_{L}$, where $J \subset K \subset L \subseteq I,|K \backslash J|=2$, $|L \backslash K|=1$ or 2 , and $J$ and $K$ have the property that all connected components are even.

If $|L \backslash K|=1$ and $L=I$, then $W_{K}$ and $W^{\prime \prime}$ have corank 1 in $W$, and so must be maximal in $\Pi_{W}^{(2)}$. If $|L \backslash K|=1$ and $L \neq I$, let $i \in I \backslash L$ be any vertex adjacent to the connected component of $L$ which contains the single vertex of $L \backslash K$. Then $L \cup\{i\}$ has all connected components even, and $W_{L \cup\{i\}}$ is the required cover.

If $|L \backslash K|=2$, we must show that $L$ itself has the property that all connected components are even. Suppose for a contradiction that it did not. Then it must have exactly two odd connected components, $L_{1}$ and $L_{2}$, containing the two elements $l_{1}$ and $l_{2}$ respectively of $L \backslash K$. Clearly the two elements of $K \backslash J$ are contained in the same connected 
component of $K$, so (swapping $L_{1}$ and $L_{2}$ if necessary) we can assume that $L_{1} \cap J=L_{1} \cap K=L_{1} \backslash\left\{l_{1}\right\}$. Now $W^{\prime \prime}$ is a parabolic subgroup of $W_{L}$, hence a product of parabolic subgroups of the irreducible components of $W_{L}$. The component of $W^{\prime \prime}$ inside $W_{L_{1}}$ must contain $W_{L_{1} \cap J}$ and cannot have odd rank, so it equals $W_{L_{1} \cap J}$, which is also the component of $W_{K}$ inside $W_{L_{1}}$. This contradicts the assumption that $\left\langle W_{K}, W^{\prime \prime}\right\rangle=W_{L}$.

Finally we prove (3). Since the product of two Cohen-Macaulay posets with minimum elements is again Cohen-Macaulay (see [16, Theorem 5.1.5 and Exercise 5.1.6]), we may assume that $W$ is irreducible. If $\operatorname{rk}(W)$ is even, then $\Pi_{W}^{(2)}$ is a pure bounded finite poset which is totally semimodular, because every lower order ideal $\left[\hat{0}, W^{\prime}\right]$ in $\Pi_{W}^{(2)}$ is isomorphic to the product of posets $\Pi_{W^{\prime \prime}}^{(2)}$ where $W^{\prime \prime}$ is irreducible of even rank. So $\Pi_{W}^{(2)}$ satisfies the Björner-Wachs criterion for being Cohen-Macaulay (see [16, Theorems 4.2.2 and 4.2.3]). If $\operatorname{rk}(W)$ is odd, the same criterion shows that $\Pi_{W}^{(2)} \cup\{\hat{1}\}$ is Cohen-Macaulay, which implies that $\Pi_{W}^{(2)}$ is.

Thus in the Coxeter case, Theorem 1.1 takes the pleasing form:

Theorem 1.6. For all $i$, there is an isomorphism of $\mathbb{Q}$-vector spaces

$$
H^{i}\left(\bar{Y}_{W}(\mathbb{R}) ; \mathbb{Q}\right) \cong \bigoplus_{\substack{W^{\prime} \in \Pi_{W}^{(2)} \\ \operatorname{rk}\left(W^{\prime}\right)=2 i}} H_{i}\left(\hat{0}, W^{\prime}\right) \otimes \operatorname{or}\left(\left(V^{W^{\prime}}\right)^{\perp}\right)
$$

which is equivariant for $N_{G L(V)}(W)$.

Proof. By Cohen-Macaulayness, the only poset homologies which survive on the right-hand side of Theorem 1.1 are those where $\operatorname{dim} A-i=$ $\operatorname{rk}_{\Pi_{\mathcal{G}}^{(2)}}(A)$, or in other words $i=\operatorname{rk}\left(W^{\prime}\right)-\operatorname{rk}_{\Pi_{W}^{(2)}}\left(W^{\prime}\right)=\frac{\operatorname{rk}\left(W^{\prime}\right)}{2}$.

Note that if it were not for the twisting by $\operatorname{or}\left(\left(V^{W^{\prime}}\right)^{\perp}\right)$, the right-hand side of Theorem 1.6 would be the $i$ th Whitney homology $W H_{i}\left(\Pi_{W}^{(2)}\right)$.

The $i=1$ special case of Theorem 1.6 is simpler, since $H_{1}\left(\hat{0}, W^{\prime}\right)$ is canonically isomorphic to $\mathbb{Q}$ when $W^{\prime}$ is an atom of the poset. Hence we have an isomorphism of representations of $N_{G L(V)}(W)$ :

$$
H^{1}\left(\bar{Y}_{W}(\mathbb{R}) ; \mathbb{Q}\right) \cong \bigoplus_{W^{\prime}} \operatorname{Ind}_{N_{G L(V)}\left(W^{\prime}\right) \cap N_{G L(V)}(W)}^{N_{G(V)}(W)}\left(\operatorname{or}\left(\left(V^{W^{\prime}}\right)^{\perp}\right)\right),
$$

where we sum over a set of representatives of the $N_{G L(V)}(W)$-conjugacy classes of irreducible rank-2 parabolic subgroups of $W$. In the classical 
types this implies the following isomorphisms:

$$
\begin{aligned}
& H^{1}\left(\bar{Y}_{A_{n}}(\mathbb{R}) ; \mathbb{Q}\right) \cong \operatorname{Ind}_{S_{n-2} \times S_{3}}^{S_{n+1}}(1 \otimes \varepsilon), \\
& H^{1}\left(\bar{Y}_{B_{n}}(\mathbb{R}) ; \mathbb{Q}\right) \cong \operatorname{Ind}_{W\left(B_{n-2}\right) \times W\left(B_{2}\right)}^{W\left(B_{n}\right)}(1 \otimes \varepsilon) \\
& \qquad \oplus \operatorname{Ind}_{W\left(B_{n-3}\right) \times\{ \pm 1\} \times S_{3}}^{W\left(B_{n}\right)}(1 \otimes 1 \otimes \varepsilon), \\
& H^{1}\left(\bar{Y}_{D_{n}}(\mathbb{R}) ; \mathbb{Q}\right) \cong \operatorname{Ind}_{W\left(B_{n-3}\right) \times\{ \pm 1\} \times S_{3}}^{W\left(B_{n}\right)}(1 \otimes 1 \otimes \varepsilon) .
\end{aligned}
$$

To describe the higher cohomology groups, we need further information about the homology of the poset $\Pi_{W}^{(2)}$; this will be the focus of the next two sections.

To save repetition, we note a close connection between the Whitney homology of $\Pi_{B_{n}}^{(2)}$ and $\Pi_{D_{n}}^{(2)}$, which should be compared with the relationship between the Whitney homology of the lattices $\Pi_{B_{n}}$ and $\Pi_{D_{n}}$ (see [10, Theorem 5.4]). Use the standard interpretations $B_{0}=$ empty arrangement, $B_{1}=A_{1}, D_{2}=A_{1} \times A_{1}, D_{3}=A_{3}$.

Proposition 1.7. For all $i$ and $n \geq 2$, we have an isomorphism

$$
W H_{i}\left(\Pi_{B_{n}}^{(2)}\right) \cong W H_{i}\left(\Pi_{D_{n}}^{(2)}\right) \oplus \operatorname{Ind}_{W\left(B_{n-2}\right) \times W\left(B_{2}\right)}^{W\left(B_{n}\right)}\left(W H_{i-1}\left(\Pi_{B_{n-2}}^{(2)}\right) \otimes 1\right)
$$

of representations of $W\left(B_{n}\right)$.

Proof. The general result [15, Theorem 3.2] provides a canonical isomorphism $H_{i}(0, A) \cong H_{i}^{f}(A)$ for all $A \in \Pi_{\mathcal{G}}^{(2)}$, where $H_{i}^{f}(A)$ denotes the homology of the chain complex $C_{\bullet}^{f}(A)$ of 2-divisible forests with root $A$. With the usual definition of the hyperplane arrangement $\mathcal{A}_{B_{n}}$, the building set $\mathcal{G}_{B_{n}}$ consists of the following subspaces, written using a basis $x_{1}, \cdots, x_{n}$ of $V^{*}$ :

$$
\begin{aligned}
\mathbb{R}^{I}= & \left\{\sum_{i \in I} a_{i} x_{i}\right\}, \text { for } I \subseteq\{1, \cdots, n\},|I| \geq 1, \text { and } \\
\mathbb{R}_{\varphi}^{L}= & \left\{\sum_{\ell \in L} a_{\ell} x_{\ell} \mid \sum_{\ell \in L} \varphi(\ell) a_{\ell}=0\right\}, \\
& \quad \text { for } L \subseteq\{1, \cdots, n\},|L| \geq 2, \varphi: L \rightarrow\{ \pm 1\} .
\end{aligned}
$$

The building set $\mathcal{G}_{D_{n}}$ can be identified with the subset of $\mathcal{G}_{B_{n}}$ consisting of all but the subspaces $\mathbb{R}^{I}$ where $|I| \leq 2$. From this it is clear that any 2-divisible forest for $\mathcal{G}_{D_{n}}$ is also a 2-divisible forest for $\mathcal{G}_{B_{n}}$; the only 2-divisible forests for $\mathcal{G}_{B_{n}}$ which are not of this kind are those which contain a subspace $\mathbb{R}^{\{i, j\}}$, necessarily unique. Hence we have a short exact sequence of chain complexes:

$$
0 \rightarrow C_{\bullet}^{f, D_{n}}(A) \rightarrow C_{\bullet}^{f, B_{n}}(A) \rightarrow \bigoplus_{\mathbb{R}^{\{i, j\}} \subseteq A} C_{\bullet-1}^{f, B_{n-2}}\left(A / \mathbb{R}^{\{i, j\}}\right) \rightarrow 0
$$


for all $A \in \Pi_{\mathcal{G}_{B_{n}}}^{(2)}$ (interpret $C_{\bullet}^{f, D_{n}}(A)$ as 0 if $\left.A \notin \Pi_{\mathcal{G}_{D_{n}}}^{(2)}\right)$. The corresponding long exact sequence of homology groups reduces to a short exact sequence, because of the Cohen-Macaulay property proved in Theorem 1.5, The result follows.

Note that the direct sum decomposition in Proposition 1.7 is not canonical, since the short exact sequence has no canonical splitting.

Corollary 1.8. For all $i$ and $n \geq 2$, we have an isomorphism

$$
\begin{aligned}
H^{i}\left(\bar{Y}_{B_{n}}(\mathbb{R}) ; \mathbb{Q}\right) \cong & H^{i}\left(\bar{Y}_{D_{n}}(\mathbb{R}) ; \mathbb{Q}\right) \\
& \oplus \operatorname{Ind}_{W\left(B_{n-2}\right) \times W\left(B_{2}\right)}^{W\left(B_{n}\right)}\left(H^{i-1}\left(\bar{Y}_{B_{n-2}}(\mathbb{R}) ; \mathbb{Q}\right) \otimes \varepsilon\right)
\end{aligned}
$$

of representations of $W\left(B_{n}\right)$.

Proof. This follows from Theorem 1.6, by incorporating the factors $\operatorname{or}\left(\left(V^{W^{\prime}}\right)^{\perp}\right)$ into the argument of the Proposition.

To be more concrete, for $n \geq 3$ the inclusion of $\mathcal{G}_{D_{n}}$ in $\mathcal{G}_{B_{n}}$ induces a surjective birational map $\bar{Y}_{B_{n}} \rightarrow \bar{Y}_{D_{n}}$. In fact, $\bar{Y}_{B_{n}}$ is the blow-up of $\bar{Y}_{D_{n}}$ along a collection of disjoint subvarieties, each isomorphic to $\bar{Y}\left(B_{n-2}(\mathbb{R})\right)$, indexed by the cosets $W\left(B_{n}\right) /\left(W\left(B_{n-2}\right) \times W\left(B_{2}\right)\right)$. The induced map in cohomology $H^{i}\left(\bar{Y}_{D_{n}}(\mathbb{R}) ; \mathbb{Q}\right) \rightarrow H^{i}\left(\bar{Y}_{B_{n}}(\mathbb{R}) ; \mathbb{Q}\right)$ is the $W\left(B_{n}\right)$-equivariant injection seen in Corollary 1.8 .

\section{Betti numbers And Euler CHARACTERISTiC}

In this section we neglect the action of $N_{G L(V)}(W)$, and seek to compute merely the Betti numbers and Euler characteristic of $\bar{Y}_{W}(\mathbb{R})$. We encode the Betti numbers in the Poincaré polynomial

$$
P\left(\bar{Y}_{W}(\mathbb{R}), t\right)=\sum_{i \geq 0} \operatorname{dim} H^{i}\left(\bar{Y}_{W}(\mathbb{R}) ; \mathbb{Q}\right)(-t)^{i},
$$

so that the Euler characteristic $\chi\left(\bar{Y}_{W}(\mathbb{R})\right)$ equals $P\left(\bar{Y}_{W}(\mathbb{R}), 1\right)$. As a consequence of Theorem 1.6 we have:

Proposition 2.1. The Poincaré polynomial $P\left(\bar{Y}_{W}(\mathbb{R}), t\right)$ equals the characteristic polynomial

$$
\chi\left(\Pi_{W}^{(2)}, t\right)=\sum_{W^{\prime} \in \Pi_{W}^{(2)}} \mu_{\Pi_{W}^{(2)}}\left(\hat{0}, W^{\prime}\right) t^{\mathrm{rk}\left(W^{\prime}\right) / 2},
$$

where $\mu_{\Pi_{W}^{(2)}}$ is the Möbius function. In particular, the Euler characteristic $\chi\left(\bar{Y}_{W}(\mathbb{R})\right)$ equals $\chi\left(\Pi_{W}^{(2)}, 1\right)$. 
Proof. Take dimension of both sides of Theorem 1.6, and use the fact that $\Pi_{W}^{(2)}$ is Cohen-Macaulay.

Computing $P\left(\bar{Y}_{W}(\mathbb{R}), t\right)$ is thus reduced to the purely combinatorial problem of finding $\chi\left(\Pi_{W}^{(2)}, t\right)$. Clearly this is 1 if $n=1$ and $1-t$ if $n=2$. Moreover,

$$
\chi\left(\Pi_{W}^{(2)}, 1\right)=0 \text { when } n \text { is even, }
$$

because the poset then has a maximum (correspondingly, the Euler characteristic of $\bar{Y}_{W}(\mathbb{R})$ is zero because it is an odd-dimensional compact manifold). This, and our knowledge that the coefficient of $-t$ is the number of irreducible rank-2 parabolic subgroups, is enough information to handle the exceptional types of rank $\leq 4$ :

$$
\begin{aligned}
& \chi\left(\Pi_{H_{3}}^{(2)}, t\right)=1-16 t, \\
& \chi\left(\Pi_{F_{4}}^{(2)}, t\right)=1-50 t+49 t^{2}, \\
& \chi\left(\Pi_{H_{4}}^{(2)}, t\right)=1-272 t+271 t^{2} .
\end{aligned}
$$

Using the technique of Sundaram (see [16, Theorem 4.4.1]) to simplify the calculations, one can compute:

$$
\begin{aligned}
& \chi\left(\Pi_{E_{6}}^{(2)}, t\right)=1-120 t+2739 t^{2}-2620 t^{3}, \\
& \chi\left(\Pi_{E_{7}}^{(2)}, t\right)=1-336 t+26229 t^{2}-230014 t^{3}, \\
& \chi\left(\Pi_{E_{8}}^{(2)}, t\right)=1-1120 t+332178 t^{2}-18066280 t^{3}+17735221 t^{4} .
\end{aligned}
$$

For this we used the Magma computational algebra package.

It only remains to treat the classical types. In [2] (see also [6, Corollary 3.16]) it is shown that

$$
\chi\left(\Pi_{A_{n}}^{(2)}, t\right)=\prod_{k=1}^{\left\lfloor\frac{n}{2}\right\rfloor}\left(1-(n+1-2 k)^{2} t\right),
$$

which implies that

$$
\chi\left(\Pi_{A_{n}}^{(2)}, 1\right)=\frac{(-1)^{\frac{n-1}{2}}(n+1) !(n-1) !}{2^{n}\left(\frac{n+1}{2}\right) !\left(\frac{n-1}{2}\right) !}, \text { when } n \text { is odd. }
$$

This formula for $\chi\left(\bar{Y}_{A_{n}}(\mathbb{R})\right)$ was proved topologically by Devadoss ([5], Theorem 3.2.3]).

A generating-function formula for the characteristic polynomial in type $B$ was effectively found in [10]: 
Proposition 2.2. In $\mathbb{Q}[t] \llbracket x \rrbracket$ we have

$$
\begin{aligned}
1+\frac{x}{2}+\sum_{n \geq 2} \chi & \left(\Pi_{B_{n}}^{(2)}, t\right) \frac{x^{n}}{2^{n} n !} \\
& =\operatorname{sech}\left(\frac{1}{2} \operatorname{arcsinh}\left(t^{1 / 2} x\right)\right) \exp \left(\frac{t^{-1 / 2}}{2} \operatorname{arcsinh}\left(t^{1 / 2} x\right)\right) .
\end{aligned}
$$

Proof. This is the $G=\{ \pm 1\}$ case of [10, (5.10)], with the first term on the right-hand side omitted since it corresponds to the maximum elements which are not included in $\Pi_{B_{n}}^{(2)}$.

Using the identity $\tanh \left(\frac{1}{2} \operatorname{arcsinh}(x)\right)=\frac{\left(1+x^{2}\right)^{1 / 2}-1}{x}$, we deduce that

$$
\chi\left(\Pi_{B_{n}}^{(2)}, 1\right)=\frac{(-1)^{\frac{n-1}{2}} n !(n-1) !}{\left(\frac{n+1}{2}\right) !\left(\frac{n-1}{2}\right) !}, \text { when } n \text { is odd. }
$$

The fact that $\chi\left(\bar{Y}_{B_{n}}(\mathbb{R})\right)=\frac{2^{n}}{n+1} \chi\left(\bar{Y}_{A_{n}}(\mathbb{R})\right)$ was proved topologically by Gaiffi (see [7, Theorem 6.9]).

Corollary 2.3. For $n \geq 2, \chi\left(\Pi_{B_{n}}^{(2)}, t\right)$ equals

$$
\sum_{m=0}^{\left\lfloor\frac{n}{2}\right\rfloor}\left(\begin{array}{c}
n \\
2 m
\end{array}\right) \frac{(4 m) !}{2^{2 m}(2 m+1) !}(-t)^{m} \prod_{\substack{1 \leq a \leq n-2 m-2 \\
a \equiv n \bmod 2}}\left(1-4 a^{2} t\right) .
$$

Proof. By a standard identity,

$$
\operatorname{sech}\left(\frac{1}{2} \operatorname{arcsinh}\left(t^{1 / 2} x\right)\right)=\sum_{m \geq 0} \frac{(4 m) !}{2^{2 m}(2 m+1) !}(-t)^{m} \frac{x^{2 m}}{2^{2 m}(2 m) !},
$$

and by an argument entirely analogous to [6. Proof of Corollary 3.16],

$$
\exp \left(\frac{t^{-1 / 2}}{2} \operatorname{arcsinh}\left(t^{1 / 2} x\right)\right)=\sum_{m \geq 0} \prod_{\substack{1 \leq a \leq m-2 \\ a \equiv m \bmod 2}}\left(1-4 a^{2} t\right) \frac{x^{m}}{2^{m} m !} .
$$

So the result follows from Proposition 2.2.

In contrast to type $A, \chi\left(\Pi_{B_{n}}^{(2)}, t\right)$ does not generally factorize into linear factors with integer coefficients, e.g. $\chi\left(\Pi_{B_{5}}^{(2)}, t\right)=1-50 t+289 t^{2}$.

Finally, Corollary 1.8 shows that

$$
\chi\left(\Pi_{D_{n}}^{(2)}, t\right)=\chi\left(\Pi_{B_{n}}^{(2)}, t\right)+\left(\begin{array}{l}
n \\
2
\end{array}\right) t \chi\left(\Pi_{B_{n-2}}^{(2)}, t\right),
$$


which implies that

$$
\chi\left(\Pi_{D_{n}}^{(2)}, 1\right)=\frac{(-1)^{\frac{n-1}{2}}(n-1)(7 n-17) n !(n-3) !}{8\left(\frac{n+1}{2}\right) !\left(\frac{n-1}{2}\right) !}, \text { when } n \geq 3 \text { is odd. }
$$

The fact that $\chi\left(\bar{Y}_{D_{n}}(\mathbb{R})\right)=\frac{2^{n-3}(7 n-17)}{(n+1)(n-2)} \chi\left(\bar{Y}_{A_{n}}(\mathbb{R})\right)$ was proved topologically in [1, Theorem 6.14].

\section{The $W\left(B_{n}\right)$-ACtion on the COHOMOlogy of $\bar{Y}_{B_{n}}(\mathbb{R})$}

We now return to the equivariant setting, and deduce from Theorem [1.6] a plethystic generating-function formula for the characters of $W\left(B_{n}\right)$ acting on the rational cohomology groups of $\bar{Y}_{B_{n}}(\mathbb{R})$, analogous to the type- $A$ formula given in [14]. (Because of Corollary [1.8, any information about $\bar{Y}_{B_{n}}(\mathbb{R})$ has immediate implications for $\bar{Y}_{D_{n}}(\mathbb{R})$.)

Let $\Lambda_{A}$ denote the usual $\mathbb{N}$-graded ring of symmetric functions, with scalars extended to $\mathbb{Q}$; thus $\Lambda_{A}=\mathbb{Q}\left[p_{i} \mid i \geq 1\right]$ where $p_{i}$ is the power sum function of degree $i$. The character of a representation $M$ of $S_{n}$ over $\mathbb{Q}$ is encapsulated in its Frobenius characteristic $\operatorname{ch}_{S_{n}}(M)$, which is a homogeneous element of $\Lambda_{A}$ of degree $n$. Let $\sim$ denote the ring involution on $\Lambda_{A}$ which corresponds to tensoring representations with the sign character $\varepsilon$; that is, the one satisfying $p_{i}^{\sim}=(-1)^{i-1} p_{i}$. We also need the associative operation $\circ$ on $\Lambda_{A}$ called plethysm (see [11, Chapter I, Section 8]), which satisfies $p_{i} \circ p_{j}=p_{i j}$; in particular, $p_{1}$ is the plethystic identity. Note that if $g \in \Lambda_{A}$ has only odd-degree terms, $(f \circ g)^{\sim}=f^{\sim} \circ g^{\sim}$ for all $f \in \Lambda_{A}$.

Following [11, Chapter I, Appendix B], we introduce a type- $B$ analogue $\Lambda_{B}:=\mathbb{Q}\left[x_{i}, y_{i} \mid i \geq 1\right]$, which is $\mathbb{N}$-graded by $\operatorname{setting} \operatorname{deg}\left(x_{i}\right)=$ $\operatorname{deg}\left(y_{i}\right)=i$. (In the notation of [loc. cit.], $W\left(B_{n}\right)$ is $\{ \pm 1\}<S_{n}, x_{i}$ is $p_{i}(\{1\})$ and $y_{i}$ is $p_{i}(\{-1\})$.) The Frobenius characteristic of a representation $M$ of $W\left(B_{n}\right)$ over $\mathbb{Q}$ is

$$
\operatorname{ch}_{W\left(B_{n}\right)}(M):=\frac{1}{2^{n} n !} \sum_{w \in W\left(B_{n}\right)} \operatorname{tr}(w, M) \prod_{i \geq 1} x_{i}^{a_{i}(w)} y_{i}^{b_{i}(w)}
$$

where $a_{i}(w)$ (respectively, $b_{i}(w)$ ) denotes the number of cycles in $w$ of length $i$ whose cycle-product is 1 (respectively, -1 ). This is a homogeneous element of $\Lambda_{B}$ of degree $n$. We define a ring involution $\sim$ on $\Lambda_{B}$ by the rules $x_{i}^{\sim}=(-1)^{i-1} x_{i}, y_{i}^{\sim}=(-1)^{i} y_{i}$; again this corresponds to tensoring representations with the sign character $\varepsilon$ of $W\left(B_{n}\right)$. We also have a right plethystic action $\circ: \Lambda_{B} \times \Lambda_{A} \rightarrow \Lambda_{B}$, which satisfies

$$
x_{i} \circ p_{j}=x_{i j}, y_{i} \circ p_{j}= \begin{cases}x_{i j}, & \text { if } j \text { is even, } \\ y_{i j}, & \text { if } j \text { is odd. }\end{cases}
$$


If $g \in \Lambda_{A}$ has only odd-degree terms, $(f \circ g)^{\sim}=f^{\sim} \circ g^{\sim}$ for all $f \in \Lambda_{B}$. See [9, Section 5] for some other properties of this action.

Complete $\Lambda_{A}$ and $\Lambda_{B}$ to the corresponding formal power series rings $\mathbb{A}_{A}=\mathbb{Q} \llbracket p_{i} \rrbracket$ and $\mathbb{A}_{B}=\mathbb{Q} \llbracket x_{i}, y_{i} \rrbracket$. We can extend plethysm and the plethystic action to these in the obvious way, provided that the righthand input has zero constant term. We define

$$
\begin{aligned}
\operatorname{Exp} & :=\sum_{n \geq 0} \operatorname{ch}_{S_{n}}(\mathbf{1})=\exp \left(\sum_{m \geq 1} \frac{p_{m}}{m}\right) \in \mathbb{A}_{A}, \\
\operatorname{Exp}_{B}: & : \sum_{n \geq 0} \operatorname{ch}_{W\left(B_{n}\right)}(\mathbf{1})=\exp \left(\sum_{m \geq 1} \frac{x_{m}+y_{m}}{2 m}\right) \in \mathbb{A}_{B}
\end{aligned}
$$

We also define Cosh and $\operatorname{Sinh}$ (respectively, $\operatorname{Cosh}_{B}$ and $\operatorname{Sinh}_{B}$ ) to be the sums of the even-degree and odd-degree terms of Exp (respectively, $\operatorname{Exp}_{B}$ ). Since $\operatorname{Cosh}_{B}$ has constant term 1, it has a multiplicative inverse $\operatorname{Sech}_{B} \in \mathbb{A}_{B}$, which also has only even-degree terms. Since Sinh has zero constant term, it has a plethystic inverse Arcsinh $\in \mathbb{A}_{A}$, which has only odd-degree terms.

To keep track of the grading by cohomological degree, we must work in $\mathbb{A}_{A} \otimes_{\mathbb{Q}} \mathbb{Q}\left[t, t^{-1}\right]$ and $\mathbb{A}_{B} \otimes_{\mathbb{Q}} \mathbb{Q}\left[t, t^{-1}\right]$ where $t$ is another indeterminate. We extend the plethysm and plethystic action to these rings by the rules $p_{i} \circ t=x_{i} \circ t=y_{i} \circ t=t^{i}$. Actually the formulae can be stated more conveniently if we use $\mathbb{Q}\left[t^{1 / 2}, t^{-1 / 2}\right]$ rather than just $\mathbb{Q}\left[t, t^{-1}\right]$, although all exponents of $t$ in the final result are integers.

In this notation, the generating function for the character of $S_{n}$ acting on the rational cohomology groups of $\bar{Y}_{A_{n-1}}(\mathbb{R})$ is as follows.

Theorem 3.1. [14, Theorem 3.5] In $\mathbb{A}_{A} \otimes_{\mathbb{Q}} \mathbb{Q}\left[t, t^{-1}\right]$ we have

$$
\begin{aligned}
1+p_{1}+\sum_{n \geq 2} \sum_{i \geq 0} \operatorname{ch}_{S_{n}}\left(H^{i}\left(\bar{Y}_{A_{n-1}}(\mathbb{R}) ; \mathbb{Q}\right)\right)(-t)^{i} \\
=\operatorname{Exp} \circ t^{-1 / 2} \operatorname{Arcsinh}^{\sim} \circ t^{1 / 2} p_{1} .
\end{aligned}
$$

(Here $t^{-1 / 2} \operatorname{Arcsinh}^{\sim} \circ t^{1 / 2} p_{1}$ is an element of $\mathbb{A}_{A} \otimes_{\mathbb{Q}} \mathbb{Q}\left[t, t^{-1}\right]$ because all terms of Arcsinh $\sim$ have odd degree; in fact, this is just Arcsinh ${ }^{\sim}$ with the degree- $(2 m+1)$ term multiplied by $t^{m}$.)

The type- $B$ analogue is:

Theorem 3.2. In $\mathbb{A}_{B} \otimes_{\mathbb{Q}} \mathbb{Q}\left[t, t^{-1}\right]$ we have

$$
\begin{aligned}
& 1+ \frac{x_{1}+y_{1}}{2}+\sum_{n \geq 2} \sum_{i \geq 0} \operatorname{ch}_{W\left(B_{n}\right)}\left(H^{i}\left(\bar{Y}_{B_{n}}(\mathbb{R}) ; \mathbb{Q}\right)\right)(-t)^{i} \\
& \quad=\left(\operatorname{Sech}_{B}^{\sim} \circ \operatorname{Arcsinh}^{\sim} \circ t^{1 / 2} p_{1}\right)\left(\operatorname{Exp}_{B} \circ t^{-1 / 2} \operatorname{Arcsinh}^{\sim} \circ t^{1 / 2} p_{1}\right) .
\end{aligned}
$$


Proof. By Theorem 1.6, we can identify $H^{\bullet}\left(\bar{Y}_{B_{n}}(\mathbb{R}) ; \mathbb{Q}\right)$ with the Whitney homology of the poset $\Pi_{B_{n}}^{(2)}$, twisted by the $\operatorname{or}\left(\left(V^{W^{\prime}}\right)^{\perp}\right)$ factors. Thus the proof proceeds almost identically to the proof for the untwisted Whitney homology of $Q_{n}^{1 \bmod 2}(\{ \pm 1\})$ given in [10, Section 5]. The key point there is that the lower order ideal $[\hat{0},(J, \pi)]$ is isomorphic to the poset product $Q^{1 \bmod 2}(J) \times \prod_{K \in \pi^{\prime}} \Pi^{1 \bmod 2}(K)$, where the posetvalued functors $Q^{1 \bmod 2}$ and $\Pi^{1 \bmod 2}$ are defined as in [10, Section 4], and $\pi^{\prime}$ denotes a set of representatives for the $\{ \pm 1\}$-orbits on $\pi$. So by the Künneth formula [16, second statement of Theorem 5.1.5],

$$
H_{\mathrm{rk}(J, \pi)}(\hat{0},(J, \pi)) \cong \widetilde{H}_{Q^{1 \bmod 2}}(J) \otimes \bigotimes_{K \in \pi^{\prime}} \widetilde{H}_{\Pi^{1 \bmod 2}}(K),
$$

where the graded $(\{ \pm 1\}<\mathbb{S})$-module $\widetilde{H}_{Q^{1} \text { mod } 2}$ and the graded $\mathbb{S}$-module $\widetilde{H}_{\Pi^{1} \bmod 2}$ are defined as in [10, Section 4]. The new ingredient here is $\operatorname{or}\left(\left(V^{W^{\prime}}\right)^{\perp}\right)$; it is easy to see that if $W^{\prime} \in \Pi_{B_{n}}^{(2)}$ corresponds to $(J, \pi) \in$ $Q_{n}^{1 \bmod 2}(\{ \pm 1\})$, then

$$
\operatorname{or}\left(\left(V^{W^{\prime}}\right)^{\perp}\right) \cong \varepsilon_{\{ \pm 1\}}(J) \otimes \bigotimes_{K \in \pi^{\prime}} \varepsilon(K)
$$

where $\varepsilon$ is the $\mathbb{S}$-module such that $\varepsilon(K)$ is a one-dimensional vector space on which the permutations of $K$ act via the sign character, and $\varepsilon_{\{ \pm 1\}}$ is the analogous $(\{ \pm 1\}<\mathbb{S})$-module. So we can translate Theorem 1.6 into the following equation in $\mathbb{A}_{B} \otimes_{\mathbb{Q}} \mathbb{Q}\left[t, t^{-1}\right]$ :

$$
\begin{aligned}
& 1+\frac{x_{1}+y_{1}}{2}+\sum_{n \geq 2} \sum_{i \geq 0} \operatorname{ch}_{W\left(B_{n}\right)}\left(H^{i}\left(\bar{Y}_{B_{n}}(\mathbb{R}) ; \mathbb{Q}\right)\right)(-t)^{i} \\
& \quad=\operatorname{ch}_{t}\left[\left(\widetilde{H}_{Q^{1 \bmod 2}}^{0 \bmod 2} \otimes \varepsilon_{\{ \pm 1\}}\right) \cdot\left(\mathbf{1}_{\{ \pm 1\}} \circ\left(\widetilde{H}_{\Pi^{1 \bmod 2}}^{1 \bmod 2} \otimes \varepsilon\right)\right)\right] .
\end{aligned}
$$

As well as the notation $\mathrm{ch}_{t}, \cdot$, o introduced in [10], we have here used the obvious definition of tensor-multiplying a graded $\mathbb{S}$-module (respectively, a graded $(\{ \pm 1\}<\mathbb{S}$ )-module) by the $\mathbb{S}$-module $\varepsilon$ (respectively, the $(\{ \pm 1\}<\mathbb{S})$-module $\left.\varepsilon_{\{ \pm 1\}}\right)$. Now we apply [10, Theorem 5.1] to the righthand side of (3.5), which converts it into

$$
\operatorname{ch}_{t}\left(\widetilde{H}_{Q^{1 \bmod 2}}^{0 \bmod 2}\right)^{\sim} \cdot\left(\operatorname{Exp}_{B} \circ \operatorname{ch}_{t}\left(\widetilde{H}_{\Pi^{1} \bmod 2}^{1 \bmod 2}\right)^{\sim}\right) .
$$

The $d=2$ case of [10, (5.9)], a rephrasing of [2, Theorem 4.7], says that

$$
\operatorname{ch}_{t}\left(\widetilde{H}_{\Pi^{1 \bmod 2}}^{1 \bmod 2}\right)=t^{-1 / 2} \operatorname{Arcsinh} \circ t^{1 / 2} p_{1},
$$

and the $d=2$ case of [10, Theorem 2.6] says that

$$
\operatorname{ch}_{t}\left(\widetilde{H}_{Q^{1 \bmod 2}}^{0 \bmod 2}\right)=\operatorname{Sech}_{B} \circ \operatorname{Arcsinh} \circ t^{1 / 2} p_{1} .
$$

Theorem 3.2 follows. 
The non-equivariant Proposition 2.2 can be obtained from this Theorem by setting $x_{1} \rightarrow x$ and all other $x_{i}, y_{i} \rightarrow 0$.

The two factors in the right-hand side of Theorem 3.2 have neatly separable meanings. View $W\left(B_{n}\right)$ as $\Gamma_{n} \rtimes S_{n}$ where $\Gamma_{n}=\{ \pm 1\}^{n}$, and note that for any representation $M$ of $W\left(B_{n}\right), S_{n}$ preserves the $\Gamma_{n^{-}}$ invariant subspace $M^{\Gamma_{n}}$ and also the subspace $M^{\Gamma_{n} \text {,prod }}$ on which $\Gamma_{n}$ acts via its character prod : $\left(\varepsilon_{1}, \cdots, \varepsilon_{n}\right) \mapsto \varepsilon_{1} \cdots \varepsilon_{n}$.

Proposition 3.3. In $\mathbb{A}_{A} \otimes_{\mathbb{Q}} \mathbb{Q}\left[t, t^{-1}\right]$ we have

$$
\begin{gathered}
1+p_{1}+\sum_{n \geq 2} \sum_{i \geq 0} \operatorname{ch}_{S_{n}}\left(H^{i}\left(\bar{Y}_{B_{n}}(\mathbb{R}) ; \mathbb{Q}\right)^{\Gamma_{n}}\right)(-t)^{i} \\
=\operatorname{Exp} \circ t^{-1 / 2} \operatorname{Arcsinh}^{\sim} \circ t^{1 / 2} p_{1}, \\
1+\sum_{n \geq 2} \sum_{i \geq 0} \operatorname{ch}_{S_{n}}\left(H^{i}\left(\bar{Y}_{B_{n}}(\mathbb{R}) ; \mathbb{Q}\right)^{\Gamma_{n}, \operatorname{prod}}\right)(-t)^{i} \\
=\operatorname{Sech}^{\sim} \circ \operatorname{Arcsinh}^{\sim} \circ t^{1 / 2} p_{1} .
\end{gathered}
$$

Proof. It is easy to see that for any representation $M$ of $W\left(B_{n}\right)$,

$$
\operatorname{ch}_{S_{n}}\left(M^{\Gamma_{n}}\right)=\left.\operatorname{ch}_{W\left(B_{n}\right)}(M)\right|_{x_{i}, y_{i} \rightarrow p_{i}} .
$$

So to prove the first equation we apply the substitution $x_{i}, y_{i} \rightarrow p_{i}$ to the right-hand side of Theorem 3.2 , noting that it respects the plethystic action. The first factor becomes $\left(\left.\operatorname{Sech}_{B}^{\sim}\right|_{x_{i}, y_{i} \rightarrow p_{i}}\right) \circ \operatorname{Arcsinh}^{\sim} \circ t^{1 / 2} p_{1}$, which equals 1 because

$$
\left.\operatorname{Exp}_{B}^{\sim}\right|_{x_{i}, y_{i} \rightarrow p_{i}}=\left.\operatorname{Exp}_{B}\right|_{x_{i} \rightarrow(-1)^{i-1} p_{i}, y_{i} \rightarrow(-1)^{i} p_{i}}=1,
$$

by definition of $\operatorname{Exp}_{B}$. The second factor becomes the right-hand side of the stated result, because

$$
\left.\operatorname{Exp}_{B}\right|_{x_{i}, y_{i} \rightarrow p_{i}}=\operatorname{Exp} .
$$

Similarly, for any representation $M$ of $W\left(B_{n}\right)$,

$$
\operatorname{ch}_{S_{n}}\left(M^{\Gamma_{n}, \operatorname{prod}}\right)=\left.\operatorname{ch}_{W\left(B_{n}\right)}(M)\right|_{x_{i} \rightarrow p_{i}, y_{i} \rightarrow-p_{i}} .
$$

So to prove the second equation we apply this substitution to the righthand side of Theorem 3.2. The first factor becomes

$$
\begin{aligned}
\left(\operatorname{Sech}_{B} \circ \operatorname{Arcsinh} \circ t^{1 / 2} p_{1}\right)^{\sim} & \mid x_{x_{i} \rightarrow p_{i}, y_{i} \rightarrow-p_{i}} \\
& =\left.\left(\operatorname{Sech}_{B} \circ \operatorname{Arcsinh} \circ t^{1 / 2} p_{1}\right)\right|_{x_{i}, y_{i} \rightarrow(-1)^{i-1}} p_{i} \\
& =\left(\left.\left(\operatorname{Sech}_{B} \circ \operatorname{Arcsinh} \circ t^{1 / 2} p_{1}\right)\right|_{x_{i}, y_{i} \rightarrow p_{i}}\right)^{\sim} \\
& =\left(\operatorname{Sech} \circ \operatorname{Arcsinh} \circ t^{1 / 2} p_{1}\right)^{\sim},
\end{aligned}
$$


which is the right-hand side of the stated result (in the last step we have used (3.8) again). The second factor becomes

$$
\begin{aligned}
\left(\operatorname{Exp}_{B} \circ t^{-1 / 2} \operatorname{Arcsinh}^{\sim}\right. & \left.\circ t^{1 / 2} p_{1}\right)\left.\right|_{x_{i} \rightarrow p_{i}, y_{i} \rightarrow-p_{i}} \\
& =\left.\left(\operatorname{Exp}_{B}^{\sim} \circ t^{-1 / 2} \operatorname{Arcsinh} \circ t^{1 / 2} p_{1}\right)\right|_{x_{i}, y_{i} \rightarrow(-1)^{i-1} p_{i}} \\
& =\left(\left.\left(\operatorname{Exp}_{B}^{\sim} \circ t^{-1 / 2} \operatorname{Arcsinh} \circ t^{1 / 2} p_{1}\right)\right|_{x_{i}, y_{i} \rightarrow p_{i}}\right)^{\sim} \\
& =1,
\end{aligned}
$$

where in the last step we have used (3.7) again.

Note that the first part of this Proposition, together with Theorem 3.1, implies an isomorphism of graded $S_{n}$-representations

$$
H^{\bullet}\left(\bar{Y}_{B_{n}}(\mathbb{R}) ; \mathbb{Q}\right)^{\Gamma_{n}} \cong H^{\bullet}\left(\bar{Y}_{A_{n-1}}(\mathbb{R}) ; \mathbb{Q}\right) .
$$

It is natural to suspect that this isomorphism is induced by a homotopy equivalence between $\Gamma_{n} \backslash \bar{Y}_{B_{n}}(\mathbb{R})$ (the topological quotient) and $\bar{Y}_{A_{n-1}}(\mathbb{R})$. By comparison, the formula analogous to Theorem 3.2 for the complex manifold $\bar{Y}_{B_{n}}(\mathbb{C})$, which is the $r=2$ case of $[9$, Theorem 3.4], implies an isomorphism of graded $S_{n}$-representations

$$
H^{\bullet}\left(\bar{Y}_{B_{n}}(\mathbb{C}) ; \mathbb{Q}\right)^{\Gamma_{n}} \cong H^{\bullet}\left(\bar{Y}_{A_{n}}(\mathbb{C}) ; \mathbb{Q}\right),
$$

where the right-hand side is restricted from $S_{n+1}$ to $S_{n}$.

Specializing further, we can find the occurrences of the four onedimensional characters of $W\left(B_{n}\right)$ in $H^{\bullet}\left(\bar{Y}_{B_{n}}(\mathbb{R}) ; \mathbb{Q}\right)$. For this we need a preparatory result:

Lemma 3.4. In $\mathbb{Q} \llbracket x \rrbracket$, Arcsinh $\left.\right|_{p_{i} \rightarrow x^{i}}=x-x^{3},\left.\operatorname{Arcsinh}^{\sim}\right|_{p_{i} \rightarrow x^{i}}=x$.

Proof. The first claim is proved in [2, p. 306], and the proof of the second is similar.

Let prod denote the one-dimensional character of $W\left(B_{n}\right)$ whose restriction to $\Gamma_{n}$ is prod as above, and whose restriction to $S_{n}$ is trivial.

Proposition 3.5. For $n \geq 2$, the multiplicities of the one-dimensional characters of $W\left(B_{n}\right)$ in $H^{i}\left(\bar{Y}_{B_{n}}(\mathbb{R}) ; \mathbb{Q}\right)$ are:

$$
\begin{aligned}
\left\langle H^{i}\left(\bar{Y}_{B_{n}}(\mathbb{R}) ; \mathbb{Q}\right), 1\right\rangle_{W\left(B_{n}\right)} & = \begin{cases}1, & \text { if } i=0, \\
0, & \text { otherwise, }\end{cases} \\
\left\langle H^{i}\left(\bar{Y}_{B_{n}}(\mathbb{R}) ; \mathbb{Q}\right), \varepsilon \cdot \operatorname{prod}\right\rangle_{W\left(B_{n}\right)} & = \begin{cases}1, & \text { if } n \neq \equiv 2 \bmod 3, i=\left\lfloor\frac{n}{3}\right\rfloor, \\
0, & \text { otherwise, }\end{cases} \\
\left\langle H^{i}\left(\bar{Y}_{B_{n}}(\mathbb{R}) ; \mathbb{Q}\right), \text { prod }\right\rangle_{W\left(B_{n}\right)} & =0, \\
\left\langle H^{i}\left(\bar{Y}_{B_{n}}(\mathbb{R}) ; \mathbb{Q}\right), \varepsilon\right\rangle_{W\left(B_{n}\right)} & = \begin{cases}1, & \text { if } n \equiv 0 \bmod 2, i=\frac{n}{2}, \\
0, & \text { otherwise. }\end{cases}
\end{aligned}
$$


In particular, the topological quotient $W\left(B_{n}\right) \backslash \bar{Y}_{B_{n}}(\mathbb{R})$ has the rational cohomology of a point.

Proof. For any representation $M$ of $S_{n},\left.\operatorname{ch}_{S_{n}}(M)\right|_{p_{i} \rightarrow x^{i}}=\langle M, 1\rangle_{S_{n}} x^{n}$. Now the first multiplicity in the statement is the multiplicity of the trivial character of $S_{n}$ in $H^{i}\left(\bar{Y}_{B_{n}}(\mathbb{R}) ; \mathbb{Q}\right)^{\Gamma_{n}}$; according to the first part of Proposition 3.3 , we find this by computing

$$
\begin{aligned}
\left(\operatorname{Exp} \circ t^{-1 / 2}\right. & \left.\operatorname{Arcsinh}^{\sim} \circ t^{1 / 2} p_{1}\right)\left.\right|_{p_{i} \rightarrow x^{i}} \\
& =\exp \left(\left.\sum_{m \geq 1} \frac{1}{m}\left(p_{m} \circ t^{-1 / 2} \operatorname{Arcsinh}^{\sim} \circ t^{1 / 2} p_{1}\right)\right|_{p_{i} \rightarrow x^{i}}\right) \\
& =\exp \left(\left.\sum_{m \geq 1} \frac{1}{m}\left(t^{-1 / 2} \operatorname{Arcsinh}^{\sim} \circ t^{1 / 2} p_{1}\right)\right|_{p_{i} \rightarrow x^{m i}, t \rightarrow t^{m}}\right) \\
& =\exp \left(\sum_{m \geq 1} \frac{1}{m} x^{m}\right) \\
& =1+x+x^{2}+x^{3}+\cdots,
\end{aligned}
$$

where the third equality uses Lemma 3.4. Similarly, the second multiplicity is that of the sign character of $S_{n}$ in $H^{i}\left(\bar{Y}_{B_{n}}(\mathbb{R}) ; \mathbb{Q}\right)^{\Gamma_{n}}$, which we find by computing

$$
\begin{aligned}
& \left.\left(\operatorname{Exp}^{\sim} \circ t^{-1 / 2} \operatorname{Arcsinh} \circ t^{1 / 2} p_{1}\right)\right|_{p_{i} \rightarrow x^{i}} \\
& \quad=\exp \left(\left.\sum_{m \geq 1} \frac{(-1)^{m-1}}{m}\left(p_{m} \circ t^{-1 / 2} \operatorname{Arcsinh} \circ t^{1 / 2} p_{1}\right)\right|_{p_{i} \rightarrow x^{i}}\right) \\
& \quad=\exp \left(\left.\sum_{m \geq 1} \frac{(-1)^{m-1}}{m}\left(t^{-1 / 2} \operatorname{Arcsinh} \circ t^{1 / 2} p_{1}\right)\right|_{p_{i} \rightarrow x^{m i}, t \rightarrow t^{m}}\right) \\
& \quad=\exp \left(\sum_{m \geq 1} \frac{(-1)^{m-1}}{m}\left(x^{m}-t^{m} x^{3 m}\right)\right) \\
& \quad=1+x-t x^{3}-t x^{4}+t^{2} x^{6}+t^{2} x^{7}-\cdots .
\end{aligned}
$$

To treat the second part of Proposition 3.3 similarly, note that

$$
\begin{aligned}
\left.\left(\operatorname{Exp}^{\sim} \circ \operatorname{Arcsinh}^{\sim} \circ t^{1 / 2} p_{1}\right)\right|_{p_{i} \rightarrow x^{i}} & =\exp \left(\sum_{m \geq 1} \frac{(-1)^{m-1}}{m} t^{m / 2} x^{m}\right) \\
& =1+t^{1 / 2} x
\end{aligned}
$$

implying that

$$
\left.\left(\mathrm{Sech}^{\sim} \circ \operatorname{Arcsinh}^{\sim} \circ t^{1 / 2} p_{1}\right)\right|_{p_{i} \rightarrow x^{i}}=1
$$


and

$$
\begin{aligned}
\left.\left(\operatorname{Exp} \circ \operatorname{Arcsinh} \circ t^{1 / 2} p_{1}\right)\right|_{p_{i} \rightarrow x^{i}} & =\exp \left(\sum_{m \geq 1} \frac{1}{m}\left(t^{m / 2} x^{m}-t^{3 m / 2} x^{3 m}\right)\right) \\
& =1+t^{1 / 2} x+t x^{2},
\end{aligned}
$$

implying that

$\left.\left(\right.$ Sech $\left.\circ \operatorname{Arcsinh} \circ t^{1 / 2} p_{1}\right)\right|_{p_{i} \rightarrow x^{i}}=\left(1+t x^{2}\right)^{-1}=1-t x^{2}+t^{2} x^{4}-t^{3} x^{6}+\cdots$.

The third and fourth multiplicity statements follow.

It is easy to deduce the multiplicities of one-dimensional characters of $W\left(B_{n}\right)$ and $W\left(D_{n}\right)$ in the cohomology groups of $\bar{Y}_{D_{n}}(\mathbb{R})$ :

Proposition 3.6. For $n \geq 2$, we have

$$
\begin{aligned}
\left\langle H^{i}\left(\bar{Y}_{D_{n}}(\mathbb{R}) ; \mathbb{Q}\right), 1\right\rangle_{W\left(B_{n}\right)} & = \begin{cases}1, & \text { if } i=0, \\
0, & \text { otherwise, }\end{cases} \\
\left\langle H^{i}\left(\bar{Y}_{D_{n}}(\mathbb{R}) ; \mathbb{Q}\right), \varepsilon \cdot \operatorname{prod}\right\rangle_{W\left(B_{n}\right)} & = \begin{cases}1, & \text { if } n \neq \equiv \bmod 3, i=\left\lfloor\frac{n}{3}\right\rfloor, \\
0, & \text { otherwise, }\end{cases} \\
\left\langle H^{i}\left(\bar{Y}_{D_{n}}(\mathbb{R}) ; \mathbb{Q}\right), \operatorname{prod}\right\rangle_{W\left(B_{n}\right)} & =0, \\
\left\langle H^{i}\left(\bar{Y}_{D_{n}}(\mathbb{R}) ; \mathbb{Q}\right), \varepsilon\right\rangle_{W\left(B_{n}\right)} & =0, \\
\left\langle H^{i}\left(\bar{Y}_{D_{n}}(\mathbb{R}) ; \mathbb{Q}\right), 1\right\rangle_{W\left(D_{n}\right)} & = \begin{cases}1, & \text { if } i=0, \\
0, & \text { otherwise, }\end{cases} \\
\left\langle H^{i}\left(\bar{Y}_{D_{n}}(\mathbb{R}) ; \mathbb{Q}\right), \varepsilon\right\rangle_{W\left(D_{n}\right)} & = \begin{cases}1, & \text { if } n \not \equiv 2 \bmod 3, i=\left\lfloor\frac{n}{3}\right\rfloor, \\
0, & \text { otherwise. }\end{cases}
\end{aligned}
$$

In particular, the topological quotient $W\left(D_{n}\right) \backslash \bar{Y}_{D_{n}}(\mathbb{R})$ has the rational cohomology of a point.

Proof. To obtain the first four statements, combine Proposition 3.5 and Corollary 1.8. The other two statements follow because $\operatorname{Ind}_{W\left(D_{n}\right)}^{W\left(B_{n}\right)}(1)=$ $1+\operatorname{prod}$ and $\operatorname{Ind}_{W\left(D_{n}\right)}^{W\left(B_{n}\right)}(\varepsilon)=\varepsilon+\varepsilon \cdot \operatorname{prod}$.

Another consequence of Theorem 3.2 is a generating function for the Euler character. First recall that by setting $t \rightarrow 1$ in Theorem 3.1 we obtain the following equation in $\mathbb{A}_{A}$ :

$$
1+p_{1}+\sum_{n \geq 2} \sum_{i \geq 0}(-1)^{i} \operatorname{ch}_{S_{n}}\left(H^{i}\left(\bar{Y}_{A_{n-1}}(\mathbb{R}) ; \mathbb{Q}\right)\right)=\operatorname{Exp} \circ \operatorname{Arcsinh}^{\sim}
$$

As observed in [14], the right-hand side is a power series in the indeterminates $p_{2^{l}}, l \geq 0$, only. The type- $B$ analogue is: 
Proposition 3.7. In $\mathbb{A}_{B}$ we have

$$
\begin{aligned}
& 1+\frac{x_{1}+y_{1}}{2}+\sum_{n \geq 2} \sum_{i \geq 0}(-1)^{i} \operatorname{ch}_{W\left(B_{n}\right)}\left(H^{i}\left(\bar{Y}_{B_{n}}(\mathbb{R}) ; \mathbb{Q}\right)\right) \\
& =\left(\operatorname{Sech}_{B}^{\sim} \circ \operatorname{Arcsinh}^{\sim}\right)\left(\operatorname{Exp}_{B} \circ \operatorname{Arcsinh}^{\sim}\right) \\
& =\text { harmonic mean of }\left.\left(\operatorname{Exp} \circ \operatorname{Arcsinh}^{\sim}\right)\right|_{p_{2 l} \rightarrow x_{2 l}} \\
& \text { and }\left.\left(\operatorname{Exp} \circ \operatorname{Arcsinh}^{\sim}\right)\right|_{p_{1} \rightarrow y_{1}, p_{2} \rightarrow x_{2 l}, l \geq 1} \\
& =\left(\frac{1}{2\left(1+x_{1}\right)}+\frac{1}{2\left(1+y_{1}\right)}\right)^{-1} \circ\left(\operatorname{Exp} \circ \operatorname{Arcsinh}^{\sim}-1\right) .
\end{aligned}
$$

Proof. The first expression is obtained simply by setting $t \rightarrow 1$ in Theorem 3.2. Now note that $\operatorname{Cosh}_{B}^{\sim}$ is the average of

$$
\operatorname{Exp}_{B}^{\sim}=\exp \left(\sum_{m \geq 1} \frac{(-1)^{m-1} x_{m}+(-1)^{m} y_{m}}{2 m}\right) \text { and } \exp \left(\sum_{m \geq 1} \frac{-x_{m}+y_{m}}{2 m}\right),
$$

from which it follows that $\operatorname{Cosh}_{B}^{\sim} \operatorname{Exp}_{B}^{-1}$ is the average of

$$
\exp \left(-\sum_{\substack{m \geq 2 \\ m \text { even }}} \frac{x_{m}}{m}-\sum_{\substack{m \geq 1 \\ m \text { odd }}} \frac{y_{m}}{m}\right) \text { and } \exp \left(-\sum_{m \geq 1} \frac{x_{m}}{m}\right) .
$$

So $\operatorname{Sech}_{B}^{\sim} \operatorname{Exp}_{B}$ is the harmonic mean of $\left.\operatorname{Exp}\right|_{p_{i} \rightarrow y_{i}, i \text { odd }}$ and $\left.\operatorname{Exp}\right|_{p_{i} \rightarrow x_{i}}$. From this the second expression follows, and the third expression is an obvious rewriting of that.

Although we do not know a general formula for the values of the Euler character of $\bar{Y}_{B_{n}}(\mathbb{R})$, it is at least clear from Proposition 3.7 that it vanishes on elements of $W\left(B_{n}\right)$ which include a cycle of length not a power of 2 , or a cycle of length $>1$ and cycle-product -1 .

The reason for including the third expression in Proposition 3.7 is to point out the analogy with the result for $\bar{Y}_{B_{n}}(\mathbb{C})$, which is the $r=2$ case of [9, Theorem 3.4]. Indeed, Proposition 3.7 can be proved by the same method as the latter result: essentially, one just uses the fact that Euler characteristic (in the version with compact supports) is additive with respect to the nested-set stratification of $\bar{Y}_{B_{n}}(\mathbb{R})$. The role of the series denoted $\mathcal{P}(2)$ in [9] is played by

$$
\begin{aligned}
\frac{x_{1}+y_{1}}{2}+\sum_{n \geq 2} \sum_{i \geq 0}(-1)^{i} \operatorname{ch}_{W\left(B_{n}\right)}( & \left.H_{c}^{i}\left(\mathbb{P} M_{B_{n}} ; \mathbb{Q}\right)\right) \\
= & 1-\frac{1}{2\left(1+x_{1}\right)}-\frac{1}{2\left(1+y_{1}\right)}
\end{aligned}
$$


(an easy calculation since $\mathbb{P} M_{B_{n}}$ is homotopy equivalent to $2^{n-1} n$ ! points), while the role of $\overline{\mathcal{P}}(1)$ is played by $\operatorname{Exp} \circ \operatorname{Arcsinh}^{\sim}-1$, because of (3.12). In the complex case one can distinguish the different cohomology groups by their Hodge weights, even after taking the alternating sum (the indeterminate $q$ performs this function in [9]), whereas in the real case this method gives only the Euler character and not the stronger Theorem 3.2 .

\section{Moduli Space intepretation of $\bar{Y}_{B_{n}}$}

It was noted in [4] that the variety $\bar{Y}_{A_{n}}$ is isomorphic to $\overline{\mathcal{M}_{0, n+2}}$, the moduli space of stable genus 0 curves with $n+2$ marked points. (See [12, Chapter III] for the definition and basic properties of this variety.) The map $M_{A_{n}} \rightarrow \bar{Y}_{A_{n}}$ induces an open embedding $\mathbb{P} M_{A_{n}} \hookrightarrow \bar{Y}_{A_{n}}$, whose image corresponds to the open subvariety $\mathcal{M}_{0, n+2}$ of $\overline{\mathcal{M}_{0, n+2}}$ parametrizing smooth (equivalently, irreducible) such curves. Explicitly, if we define the hyperplane arrangement of type $A_{n}$ in $\mathbb{G}_{a} \backslash \mathbb{A}^{n+1}$, where the additive group $\mathbb{G}_{a}$ acts by simultaneous translation of the coordinates, then the morphism $M_{A_{n}} \rightarrow \mathcal{M}_{0, n+2}$ sends $\left(x_{1}, \cdots, x_{n+1}\right)$ to the point parametrizing $\mathbb{P}^{1}$ with marked points $x_{1}, \cdots, x_{n+1}, \infty$.

The variety $\overline{\mathcal{M}_{0, n+2}}$ has a well-known stratification, where the strata are indexed by rooted trees with leaves labelled $1, \cdots, n+1$ : the open stratum $\mathcal{M}_{0, n+2}$ corresponds to the tree whose only vertices are the root and the leaves, and more generally the other vertices of the tree specify the pattern of intersections of the components of the stable curve (the root corresponds to the last marked point). This stratification corresponds in a natural way to the nested-set stratification of $\bar{Y}_{A_{n}}$ defined in [4].

One striking consequence of the isomorphism $\bar{Y}_{A_{n}} \cong \overline{\mathcal{M}}_{0, n+2}$ is that the action of the linear automorphism group $W\left(A_{n}\right)=S_{n+1}$ can be extended to an action of $S_{n+2}$ (permuting the marked points). The action of the 'non-linear' symmetries on cohomology is difficult to describe from the point of view of Theorem 1.6. Nevertheless, in [14 the formula for the character of $S_{n+1}$ on the rational cohomology groups of $\overline{\mathcal{M}_{0, n+2}}(\mathbb{R})$ resulting from Theorem 3.1 was extended to all of $S_{n+2}$.

Now we want to give a similar moduli space interpretation of $\bar{Y}_{B_{n}}$. Realize the hyperplane complement $M_{B_{n}}$ in the usual way:

$$
M_{B_{n}}=\left\{\left(x_{1}, \cdots, x_{n}\right) \in \mathbb{A}^{n} \mid x_{i} \neq 0 \text { for all } i, x_{i} \neq \pm x_{j} \text { for } i \neq j\right\} .
$$

We can then define a closed embedding $\pi: M_{B_{n}} \rightarrow M_{A_{2 n}}$ by

$$
\pi\left(x_{1}, \cdots, x_{n}\right)=\left(x_{1}, \cdots, x_{n},-x_{1}, \cdots,-x_{n}, 0\right),
$$


where the right-hand side represents a $\mathbb{G}_{a}$-orbit. Clearly this embedding descends to an embedding $\pi: \mathbb{P} M_{B_{n}} \hookrightarrow \mathbb{P} M_{A_{2 n}} \cong \mathcal{M}_{0,2 n+2}$, whose image $\pi\left(\mathbb{P} M_{B_{n}}\right)$ is the closed subvariety parametrizing smooth genus 0 curves with $2 n+2$ marked points and an involution which fixes the last two marked points and interchanges the $j$ th and $(n+j)$ th for $1 \leq j \leq n$ (such an involution is clearly unique if it exists).

Theorem 4.1. There is a closed embedding $\pi: \bar{Y}_{B_{n}} \hookrightarrow \overline{\mathcal{M}_{0,2 n+2}}$ whose restriction to $\mathbb{P} M_{B_{n}}$ is the embedding $\mathbb{P} M_{B_{n}} \hookrightarrow \mathcal{M}_{0,2 n+2}$ described above. The image $\pi\left(\bar{Y}_{B_{n}}\right)$ is the subvariety parametrizing stable genus 0 curves with $2 n+2$ marked points and an involution which fixes the last two marked points, interchanges the $j$ th and $(n+j)$ th for $1 \leq j \leq n$, and does not fix any component pointwise (such an involution is unique if it exists). The stratification of $\bar{Y}_{B_{n}}$ obtained by intersecting this image with the strata of $\overline{\mathcal{M}_{0,2 n+2}}$ is the nested-set stratification of [4].

Proof. The first statement follows from the fact that $\pi$ induces a surjective map of building sets $\mathcal{G}_{A_{2 n}} \rightarrow \mathcal{G}_{B_{n}}$; see [15, Proposition 2.4 and the last statement of Corollary 2.6].

The image $\pi\left(\bar{Y}_{B_{n}}\right)$ equals the closure in $\overline{\mathcal{M}_{0,2 n+2}}$ of the subvariety $\pi\left(\mathbb{P} M_{B_{n}}\right) \subseteq \mathcal{M}_{0,2 n+2}$ described above; we must show that this equals the subvariety $X \subseteq \overline{\mathcal{M}_{0,2 n+2}}$ parametrizing stable curves with an involution as stated. That $X$ is contained in the closure of $\pi\left(\mathbb{P} M_{B_{n}}\right)$ follows from the fact that every stable genus 0 curve with $2 n+2$ marked points and an involution of the stated form can be obtained by progressively degenerating a smooth one (maintaining the existence of an involution of the stated form throughout the degeneration). For example:

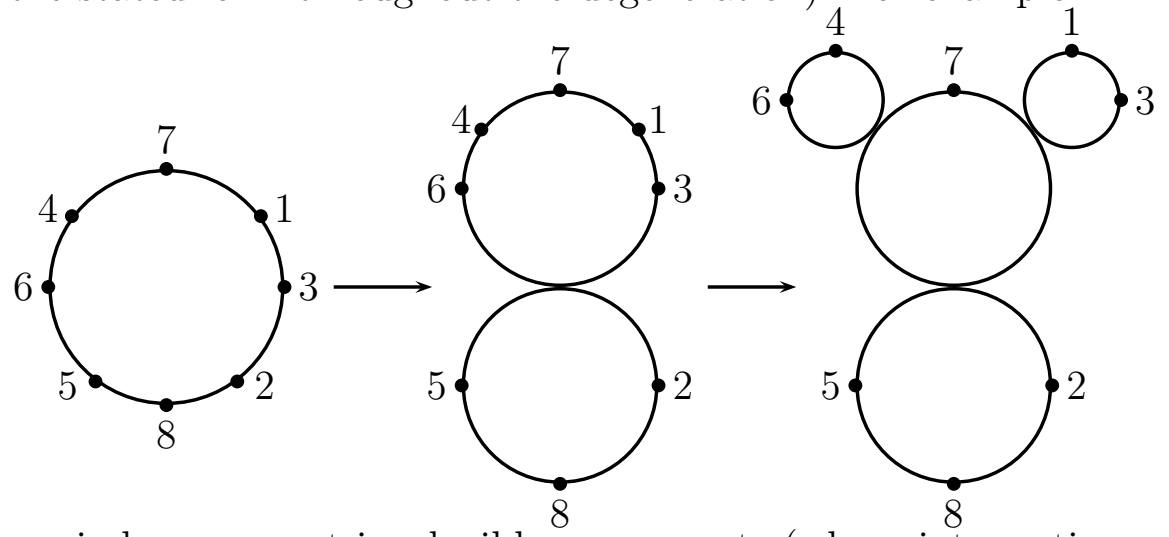

Here circles represent irreducible components (whose intersections are transverse, despite appearances), and arrows indicate a passage to the limit in some family of curves; the claim is just that the vertical axis of symmetry can be maintained throughout. 
Now take any point $x_{C}$ in the closure of $\pi\left(\mathbb{P} M_{B_{n}}\right)$, corresponding to the isomorphism class of a stable genus 0 curve $C$ with $2 n+2$ marked points; since the existence of an involution fixing the last two marked points and interchanging the $j$ th and $(n+j)$ th for $1 \leq j \leq n$ is a closed property, $C$ has such an involution, say $\tau$. We must show that $\tau$ does not fix any component of $C$ pointwise. Suppose that it did; it is easy to see that there must be some pointwise-fixed component $C_{1}$ such that the point in $C_{1}$ 'closest to' the $(2 n+1)$ th marked point is different from the point in $C_{1}$ 'closest to' the $(2 n+2)$ th marked point. By stability, $C_{1}$ must have at least one other special point, which is the point in $C_{1}$ 'closest to' the $j$ th marked point and hence also the $(n+j)$ th marked point for some $1 \leq j \leq n$. For example, suppose $n=3$ and $C$ is:

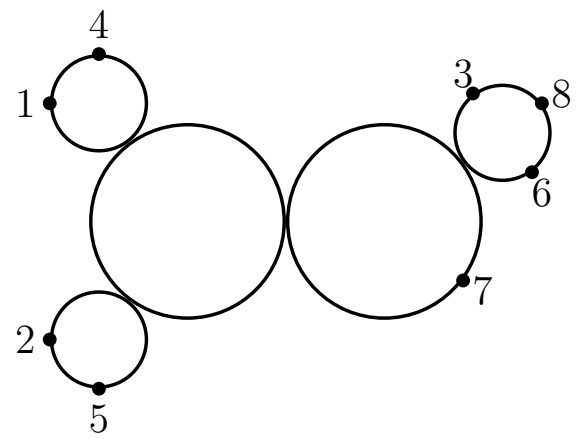

Here all components are fixed by $\tau$, but only the two represented by larger circles are pointwise-fixed. Of these, only the right-hand one satisfies the condition of $C_{1}$. We can take either $j=1$ or $j=2$, but not $j=3$.

Now there is a projective morphism $p_{j}: \overline{\mathcal{M}_{0,2 n+2}} \rightarrow \overline{\mathcal{M}_{0,4}} \cong \mathbb{P}^{1}$ defined by 'stably forgetting' all marked points except the $j$ th, $(n+j)$ th, $(2 n+1)$ th and $(2 n+2)$ th, and what we know about $C_{1}$ implies that $x_{C}$ belongs to the preimage $p_{j}^{-1}(\{b\})$, where $b$ is one of the three points of $\overline{\mathcal{M}_{0,4}} \backslash \mathcal{M}_{0,4}$. On the other hand, $\pi\left(\mathbb{P} M_{B_{n}}\right)$ is contained in the preimage $p_{j}^{-1}(\{a\})$ where $a \in \mathcal{M}_{0,4}$ parametrizes the curve $\mathbb{P}^{1}$ with marked points $1,-1,0, \infty$. This contradicts the assumption that $x_{C}$ was in the closure of $\pi\left(\mathbb{P} M_{B_{n}}\right)$.

Finally, it is easy to see that a stratum of $\overline{\mathcal{M}_{0,2 n+2}}$ intersects the subvariety $\pi\left(\bar{Y}_{B_{n}}\right)$ if and only if the corresponding tree admits an involution which fixes the root and the $(2 n+1)$ th leaf, interchanges the $j$ th and $(n+j)$ th leaves for $1 \leq j \leq n$, and does not fix any edges except those forming the path from the root to the $(2 n+1)$ th leaf (such an involution is unique if it exists). This set of trees can be easily identified with the set denoted $\mathcal{T}(2, n)$ in [9, Section 6], and the final statement follows from what was said there. 
One corollary is that the sequence of varieties $\left(\bar{Y}_{B_{n}}\right)$ form a right module for the operad $\left(\overline{\mathcal{M}_{0, n}}\right)$; for $1 \leq j \leq n$, the operation

$$
\circ_{j}: \bar{Y}_{B_{n}} \times \overline{\mathcal{M}}_{0, m+1} \rightarrow \bar{Y}_{B_{n+m-1}}
$$

is defined by gluing two copies of the second stable curve to the first, at the $j$ th and $(n+j)$ th marked points, and renumbering marked points appropriately. A similar construction is made in [1].

Observe that if $C$ is a stable genus 0 curve with $2 n+2$ marked points and an involution $\tau$ as in Theorem 4.1, then the fixed points of $\tau$ are exactly the last two marked points and the finite set of nodes which lie on the 'path' between them. The last marked point is thus determined by the rest of the data, and $C$ is still stable without it, so $\bar{Y}_{B_{n}}$ can also be identified with a subvariety of $\overline{\mathcal{M}_{0,2 n+1}}$. Indeed, the above proof would have worked just as well for the embedding $M_{B_{n}} \hookrightarrow M_{A_{2 n-1}}$ obtained by removing the last coordinate of $\pi: M_{B_{n}} \hookrightarrow M_{A_{2 n}}$.

The advantage of the description in Theorem 4.1 is that it makes clear some extra symmetry. The subgroup of $S_{2 n+2}$ which preserves $\pi\left(\bar{Y}_{B_{n}}\right)$ is the product $W\left(B_{n}\right) \times\langle(2 n+1,2 n+2)\rangle$, where $W\left(B_{n}\right)$ is embedded in $S_{2 n}$ as the centralizer of $j \leftrightarrow n+j$. The resulting action of $W\left(B_{n}\right)$ on $\bar{Y}_{B_{n}}$ is the one we have been considering throughout the paper; the action of $(2 n+1,2 n+2)$ gives an involution $\sigma$ of $\bar{Y}_{B_{n}}$ which commutes with the $W\left(B_{n}\right)$-action. Note that the restriction of $\sigma$ to $\mathbb{P} M_{B_{n}}$ is the map $\left(x_{1}, \cdots, x_{n}\right) \mapsto\left(x_{1}^{-1}, \cdots, x_{n}^{-1}\right)$.

Proposition 4.2. The fixed subvariety $\left(\bar{Y}_{B_{n}}\right)^{\sigma}$ is isomorphic to the disjoint union of $2^{n-1}$ copies of $\overline{\mathcal{M}_{0, n+1}}$.

Proof. Suppose that $x_{C} \in \pi\left(\bar{Y}_{B_{n}}\right)$ corresponds to the isomorphism class of a stable curve $C$ with involution $\tau$ as above. Then $\sigma\left(x_{C}\right)=x_{C}$ if and only if $C$ has another involution $\tau^{\prime}$ which fixes the first $2 n$ marked points and interchanges the last two. This implies that the last two marked points lie on the same component $C_{1}$, since otherwise there would be another marked point which was 'closer to' one than to the other. It is also clear that $C_{1}$ has exactly two other special points $a$ and $\tau(a)$, which are the fixed points of $\tau^{\prime}$ in $C_{1}$. If $C^{\prime}$ denotes the union of the components $\neq C_{1}$ to which $a$ is the closest point of $C_{1}$, then $C^{\prime}$ is a stable curve with $n+1$ marked points (the $n$ of the original $2 n+2$ which it contains, together with $a$ ). Since the division of the first $2 n$ marked points into two parts interchanged by $\tau$ can be made in $2^{n-1}$ ways, the result follows. 
Corollary 4.3. The Lefschetz number $\sum_{i}(-1)^{i} \operatorname{tr}\left(\sigma, H^{i}\left(\bar{Y}_{B_{n}}(\mathbb{R}) ; \mathbb{Q}\right)\right)$ equals 0 if $n$ is odd, and

$$
\frac{(-1)^{\frac{n-2}{2}} n !(n-2) !}{\left(\frac{n}{2}\right) !\left(\frac{n-2}{2}\right) !} \text { if } n \text { is even. }
$$

Proof. By a well-known principle (see [17], for instance), the Lefschetz number is $\chi\left(\bar{Y}_{B_{n}}(\mathbb{R})^{\sigma}\right)$, which equals $2^{n-1} \chi\left(\bar{Y}_{A_{n-1}}(\mathbb{R})\right)$ by the Proposition. Thus (2.5) gives the result.

One could perhaps determine $\sum_{i}(-1)^{i} \operatorname{tr}\left(w \sigma, H^{i}\left(\bar{Y}_{B_{n}}(\mathbb{R}) ; \mathbb{Q}\right)\right)$ for general $w \in W\left(B_{n}\right)$ by similar arguments.

We can describe the action of $\sigma$ on $H^{1}\left(\bar{Y}_{B_{n}}(\mathbb{R}) ; \mathbb{Q}\right)$ using an explicit basis. Theorem 1.6 showed that $H^{1}\left(\bar{Y}_{B_{n}}(\mathbb{R}) ; \mathbb{Q}\right)$ is canonically the direct sum of one-dimensional subspaces corresponding to the irreducible rank-2 parabolic subgroups of $W\left(B_{n}\right)$, or equivalently to those factors in the right-hand side of (1.1) which are isomorphic to $\mathbb{P}^{1}$. More precisely, one can construct a basis using the projections to these factors, as follows (recall the notation for the building set $\mathcal{G}_{B_{n}}$ introduced in the proof of Proposition [1.7). For any $i \neq j$ in $\{1, \cdots, n\}$, define a morphism $\rho_{i j}: \bar{Y}_{B_{n}} \rightarrow \mathbb{P}^{1}$ as the composition of the projection $\bar{Y}_{B_{n}} \rightarrow$ $\mathbb{P}\left(V /\left(\mathbb{R}^{\{i, j\}}\right)^{\perp}\right)$ and the identification $\mathbb{P}\left(V /\left(\mathbb{R}^{\{i, j\}}\right)^{\perp}\right) \cong \mathbb{P}^{1}$ which takes $x_{i}$ and $x_{j}$ as the homogeneous coordinates, in that order. Thus the restriction of $\rho_{i j}$ to $\mathbb{P} M_{B_{n}}$ is the map $\left(x_{1}, \cdots, x_{n}\right) \mapsto x_{i} x_{j}^{-1}$. Denote by $\nu_{i j}$ the pull-back $\rho_{i j}^{*}(\alpha) \in H^{1}\left(\bar{Y}_{B_{n}}(\mathbb{R}) ; \mathbb{Q}\right)$, where $\alpha \in H^{1}\left(\mathbb{P}^{1}(\mathbb{R}) ; \mathbb{Q}\right)$ is the standard class. For any distinct $i, j, k$ in $\{1, \cdots, n\}$ and $\varphi:\{i, j, k\} \rightarrow$ $\{ \pm 1\}$, define $\rho_{i j k, \varphi}: \bar{Y}_{B_{n}} \rightarrow \mathbb{P}^{1}$ as the composition of the projection $\bar{Y}_{B_{n}} \rightarrow \mathbb{P}\left(V /\left(\mathbb{R}_{\varphi}^{\{i, j, k\}}\right)^{\perp}\right)$ and the identification $\mathbb{P}\left(V /\left(\mathbb{R}_{\varphi}^{\{i, j, k\}}\right)^{\perp}\right) \cong \mathbb{P}^{1}$ which takes $\varphi(i) x_{i}-\varphi(k) x_{k}$ and $\varphi(j) x_{j}-\varphi(k) x_{k}$ as the homogeneous coordinates, in that order. Thus the restriction of $\rho_{i j k, \varphi}$ to $\mathbb{P} M_{B_{n}}$ is the $\operatorname{map}\left(x_{1}, \cdots, x_{n}\right) \mapsto\left(\varphi(i) x_{i}-\varphi(k) x_{k}\right)\left(\varphi(j) x_{j}-\varphi(k) x_{k}\right)^{-1}$. Denote by $\omega_{i j k, \varphi}$ the pull-back $\rho_{i j k, \varphi}^{*}(\alpha) \in H^{1}\left(\bar{Y}_{B_{n}}(\mathbb{R}) ; \mathbb{Q}\right)$.

Proposition 4.4. (1) $\nu_{j i}=-\nu_{i j}, \omega_{i j k,-\varphi}=\omega_{i j k, \varphi}$, and

$$
\omega_{i_{w(1)} i_{w(2)} i_{w(3)}, \varphi}=\varepsilon(w) \omega_{i_{1} i_{2} i_{3}, \varphi} \text { for } w \in S_{3} .
$$

(2) For any element $\left(\varepsilon_{1}, \cdots, \varepsilon_{n}\right)$ w of $W\left(B_{n}\right)$, where $\left(\varepsilon_{1}, \cdots, \varepsilon_{n}\right) \in$ $\Gamma_{n}$ and $w \in S_{n}$,

$$
\begin{aligned}
\left(\varepsilon_{1}, \cdots, \varepsilon_{n}\right) w \cdot \nu_{i j} & =\varepsilon_{w(i)} \varepsilon_{w(j)} \nu_{w(i) w(j)}, \\
\left(\varepsilon_{1}, \cdots, \varepsilon_{n}\right) w \cdot \omega_{i j k, \varphi} & =\omega_{w(i) w(j) w(k),\left(w(a) \mapsto \varepsilon_{w(a)} \varphi(a)\right)} .
\end{aligned}
$$


(3) The disjoint union of $\left\{\nu_{i j} \mid 1 \leq i<j \leq n\right\}$ and $\left\{\omega_{i j k, \varphi} \mid 1 \leq i<\right.$ $j<k \leq n\}$, where to avoid redundancy we take only one from each pair $\pm \varphi$, is a basis of $H^{1}\left(\bar{Y}_{B_{n}}(\mathbb{R}) ; \mathbb{Q}\right)$.

(4) The span of $\left\{\omega_{i j k, \varphi}\right\}$ is the image of the natural injective map $H^{1}\left(\bar{Y}_{D_{n}}(\mathbb{R}) ; \mathbb{Q}\right) \rightarrow H^{1}\left(\bar{Y}_{B_{n}}(\mathbb{R}) ; \mathbb{Q}\right)$ mentioned in Section 1.

(5) The span of $\left\{\nu_{i j}\right\}$ is the $(-1)$-eigenspace of $\sigma$ on $H^{1}\left(\bar{Y}_{B_{n}}(\mathbb{R}) ; \mathbb{Q}\right)$.

(6) $\sigma\left(\omega_{i j k, \varphi}\right)=\omega_{i j k, \varphi}-\varphi(i) \varphi(j) \nu_{i j}-\varphi(j) \varphi(k) \nu_{j k}-\varphi(k) \varphi(i) \nu_{k i}$.

Proof. Parts (1) and (2) follow easily from the definitions, and parts (3) and (4) from the construction of the isomorphism in [15, Theorem 3.7]. For parts (5) and (6), we use the embedding $\pi: \bar{Y}_{B_{n}} \hookrightarrow \overline{\mathcal{M}}_{0,2 n+2}$. Recall the elements $\omega_{i j k l} \in H^{1}\left(\overline{\mathcal{M}_{0,2 n+2}}(\mathbb{R}) ; \mathbb{Q}\right)$ from [6], defined as $\rho_{i j k l}^{*}(\alpha)$ for certain morphisms $\rho_{i j k l}: \overline{\mathcal{M}_{0,2 n+2}} \rightarrow \mathbb{P}^{1}$, where $i, j, k, l$ are distinct elements of $\{1, \cdots, 2 n+2\}$. It is clear from the definitions that the morphism $\rho_{i j}$ used above is the composition $\rho_{i, j, 2 n+1,2 n+2} \circ \pi$, and the morphism $\rho_{i j k, \varphi}$ is the composition $\rho_{i^{\varphi}, j^{\varphi}, k^{\varphi}, 2 n+2} \circ \pi$, where $i^{\varphi}$ denotes $i$ if $\varphi(i)=1$ and $i+n$ if $\varphi(i)=-1$. Hence

$$
\nu_{i j}=\pi^{*}\left(\omega_{i, j, 2 n+1,2 n+2}\right), \omega_{i j k, \varphi}=\pi^{*}\left(\omega_{i^{\varphi}, j^{\varphi}, k^{\varphi}, 2 n+2}\right) .
$$

Using the definition of $\sigma$ and the relation [6, (2.1)], we calculate

$$
\begin{aligned}
\sigma\left(\nu_{i j}\right)= & \pi^{*}\left(\omega_{i, j, 2 n+2,2 n+1}\right)=\pi^{*}\left(-\omega_{i, j, 2 n+1,2 n+2}\right)=-\nu_{i j}, \\
\sigma\left(\omega_{i j k, \varphi}\right)= & \pi^{*}\left(\omega_{i^{\varphi}, j^{\varphi}, k^{\varphi}, 2 n+1}\right) \\
= & \pi^{*}\left(\omega_{i^{\varphi}, j^{\varphi}, k^{\varphi}, 2 n+2}\right. \\
& \left.\quad-\omega_{i^{\varphi}, j^{\varphi}, 2 n+1,2 n+2}-\omega_{j^{\varphi}, k^{\varphi}, 2 n+1,2 n+2}-\omega_{k^{\varphi}, i^{\varphi}, 2 n+1,2 n+2}\right) \\
= & \omega_{i j k, \varphi}-\varphi(i) \varphi(j) \nu_{i j}-\varphi(j) \varphi(k) \nu_{j k}-\varphi(k) \varphi(i) \nu_{k i} .
\end{aligned}
$$

Thus part (6) is proved, and the span of $\left\{\nu_{i j}\right\}$ is at least contained in the $(-1)$-eigenspace of $\sigma$; however, (6) shows that $\sigma$ acts trivially on the quotient by the span of $\left\{\nu_{i j}\right\}$, which proves (5).

The fixed point subspace $H^{1}\left(\bar{Y}_{B_{n}}(\mathbb{R}) ; \mathbb{Q}\right)^{\sigma}$ is spanned by the elements

$$
\widetilde{\omega}_{i j k, \varphi}=\omega_{i j k, \varphi}-\frac{1}{2} \varphi(i) \varphi(j) \nu_{i j}-\frac{1}{2} \varphi(j) \varphi(k) \nu_{j k}-\frac{1}{2} \varphi(k) \varphi(i) \nu_{k i},
$$

and is isomorphic to $H^{1}\left(\bar{Y}_{D_{n}}(\mathbb{R}) ; \mathbb{Q}\right)$ as a representation of $W\left(B_{n}\right)$ via the map $\widetilde{\omega}_{i j k, \varphi} \mapsto \omega_{i j k, \varphi}$. However, this isomorphism does not generalize to higher-degree cohomologies (indeed, Corollary 4.3 shows that $\operatorname{dim} H^{2}\left(\bar{Y}_{B_{4}}(\mathbb{R}) ; \mathbb{Q}\right)^{\sigma}=3$, whereas $\left.\operatorname{dim} H^{2}\left(\bar{Y}_{D_{4}}(\mathbb{R}) ; \mathbb{Q}\right)=15\right)$.

We conjecture that the cohomology ring $H^{\bullet}\left(\bar{Y}_{B_{n}}(\mathbb{R}) ; \mathbb{Q}\right)$ is generated in degree 1 . Since the analogous statement for $\overline{M_{0, n}}(\mathbb{R})$ was proved in [6], it is equivalent to conjecture that the homomorphism $\pi^{*}: H^{\bullet}\left(\overline{M_{0,2 n+2}}(\mathbb{R}) ; \mathbb{Q}\right) \rightarrow H^{\bullet}\left(\bar{Y}_{B_{n}}(\mathbb{R}) ; \mathbb{Q}\right)$ is surjective. Calculations 
for small $n$ suggest that the only relations satisfied by the above basis elements of $H^{1}\left(\bar{Y}_{B_{n}}(\mathbb{R}) ; \mathbb{Q}\right)$ are those forced by the relations in [6, Definition 2.1]; more precisely, that the ideal $\operatorname{ker}\left(\pi^{*}\right)$ of $H^{\bullet}\left(\overline{M_{0,2 n+2}}(\mathbb{R}) ; \mathbb{Q}\right)$ is also generated in degree 1.

\section{REFERENCES}

[1] S. M. Armstrong, M. Carr, S. L. Devadoss, E. Engler, A. Leininger, And M. Manapat, Point configurations and Coxeter operads, math.GT/0502159

[2] A. R. Calderbank, P. Hanlon, and R. W. Robinson, Partitions into even and odd block size and some unusual characters of the symmetric groups, Proc. London Math. Soc. (3), 53 (1986), pp. 288-320.

[3] M. Davis, T. Januszkiewicz, And R. Scott, Fundamental groups of blowups, Advances in Math., 177 (2003), pp. 115-179.

[4] C. De Concini and C. Procesi, Wonderful models of subspace arrangements, Selecta Math. (N.S.), 1 (1995), pp. 459-494.

[5] S. L. Devadoss, Tessellations of moduli spaces and the mosaic operad, in Homotopy Invariant Algebraic Structures (Baltimore, MD, 1998), Contemp. Math., 239, Amer. Math. Soc., Providence, RI, 1999, pp. 91-114.

[6] P. Etingof, A. Henriques, J. Kamnitzer, And E. Rains, The cohomology ring of the real locus of the moduli space of stable genus 0 curves with marked points, math.AT/0507514.

[7] G. GaIfFI, Real structures of models of arrangements, Int. Math. Res. Not., 2004:64 (2004), pp. 3439-3467.

[8] V. Ginzburg and M. Kapranov, Koszul duality for operads, Duke Math. J., 76 (1994), pp. 203-272.

[9] A. Henderson, Representations of wreath products on cohomology of De Concini-Procesi compactifications, Int. Math. Res. Not., 2004:20 (2004), pp. $983-1021$.

[10] A. Henderson, Plethysm for wreath products and homology of sub-posets of Dowling lattices, Electron. J. Combin., 13 (2006), no. 1, Research Paper 87, $25 \mathrm{pp}$.

[11] I. G. Macdonald, Symmetric Functions and Hall Polynomials, second edition, Oxford University Press, 1995.

[12] Y. I. Manin, Frobenius Manifolds, Quantum Cohomology, and Moduli Spaces, vol. 47 of American Mathematical Society Colloquium Publications, American Mathematical Society, 1999.

[13] P. Orlik and H. Terao, Arrangements of Hyperplanes, Springer-Verlag, 1992.

[14] E. RAINs, The action of $S_{n}$ on the cohomology of $\overline{M_{0, n}}(\mathbb{R})$, math.AT/0601573.

[15] E. RAIns, The homology of real subspace arrangements, math.AT/0610743.

[16] M. L. WACHS, Poset topology: tools and applications, math.CO/0602226, to appear in Geometric Combinatorics, IAS/Park City Mathematics Series, American Mathematical Society.

[17] C. T. C. WALL, A note on symmetry of singularities, Bull. London Math. Soc., 12 (1980), pp. 169-175. 
[18] S. Yuzvinsky, Cohomology bases for the De Concini-Procesi models of hyperplane arrangements and sums over trees, Invent. Math., 127 (1997), pp. 319 335 .

School of Mathematics and Statistics, University of Sydney, NSW 2006, AUSTRALIA

E-mail address: anthonyh@maths.usyd.edu.au

Department of Mathematics, California Institute of Technology, PASAdena, CA 91125, USA

E-mail address: rains@caltech.edu 